\title{
T-dualization of type II superstring theory in double space
}

\author{
B. Nikolića ${ }^{\text {, B. Sazdović }}{ }^{\mathrm{b}}$ \\ Institute of Physics Belgrade, University of Belgrade, Pregrevica 118, Belgrade, Serbia
}

Received: 12 October 2016/ Accepted: 14 March 2017 / Published online: 27 March 2017

(C) The Author(s) 2017. This article is an open access publication

\begin{abstract}
In this article we offer a new interpretation of the T-dualization procedure of type II superstring theory in the double space framework. We use the ghost free action of type II superstring in pure spinor formulation in approximation of constant background fields up to the quadratic terms. Tdualization along any subset of the initial coordinates, $x^{a}$, is equivalent to the permutation of this subset with subset of the corresponding T-dual coordinates, $y_{a}$, in double space coordinate $Z^{M}=\left(x^{\mu}, y_{\mu}\right)$. Requiring that the T-dual transformation law after the exchange $x^{a} \leftrightarrow y_{a}$ has the same form as the initial one, we obtain the T-dual NS-NS and NS-R background fields. The T-dual R-R field strength is determined up to one arbitrary constant under some assumptions. The compatibility between supersymmetry and T-duality produces a change of bar spinors and R-R field strength. If we dualize an odd number of dimensions $x^{a}$, such a change flips type IIA/B to type II B/A. If we T-dualize the time-like direction, one imaginary unit $i$ maps type II superstring theories to type II ${ }^{\star}$ ones.
\end{abstract}

\section{Introduction}

T-duality is a fundamental feature of string theory [1-8]. As a consequence of T-duality there is no physical difference between string theory compactified on a circle of radius $R$ and circle of radius $1 / R$. This conclusion can be generalized to tori of various dimensions.

The mathematical realization of T-duality is given by Buscher T-dualization procedure $[4,5]$. If the background fields have global isometries along some directions then we can localize that symmetry introducing gauge fields. The next step is to add the new term in the action with Lagrange

This work is supported in part by the Serbian Ministry of Education, Science and Technological Development, under contract No. 171031.

\footnotetext{
a e-mail: bnikolic@ipb.ac.rs

be-mail: sazdovic@ipb.ac.rs
}

multipliers which forces these gauge fields to be unphysical. Finally, we can use gauge freedom to fix initial coordinates. Varying this gauge fixed action with respect to the Lagrange multipliers one gets the initial action and varying with respect to the gauge fields one gets the T-dual action.

Buscher T-dualization can be applied along directions on which background fields do not depend [4-10]. Such a procedure was used in Refs. [11-18] in the context of closed string noncommutativity. There is a generalized Buscher procedure which deals with background fields depending on all coordinates. The generalized procedure was applied to the case of bosonic string moving in the weakly curved background $[19,20]$. It leads directly to closed string noncommutativity [21].

The Buscher procedure can be considered as the definition of T-dualization. But there are also other frameworks in which we can represent T-dualization which must be in accordance with the Buscher procedure. Here we talk about the double space formalism which was the subject of the articles about 20 years ago [22-26]. Double space is spanned by coordinates $Z^{M}=\left(x^{\mu}, y_{\mu}\right)(\mu=0,1,2, \ldots, D-1)$, where $x^{\mu}$ and $y_{\mu}$ are the coordinates of the $D$-dimensional initial and T-dual space-time, respectively. Interest for this subject emerged again with Refs. [27-34], where T-duality is related with $O(d, d)$ transformations. The approach of Ref. [22] has been recently improved when the T-dualization along some subset of the initial and corresponding subset of the T-dual coordinates has been interpreted as permutation of these subsets in the double space coordinates $[35,36]$.

Let us motivate our interest in this subject. It is well known that T-duality is important feature in understanding M-theory. In fact, five consistent superstring theories are connected by a web of $\mathrm{T}$ and $\mathrm{S}$ dualities. In the beginning we are going to pay attention to the T-duality. To obtain formulation of M-theory it is not enough to find all corresponding T-dual theories. We must construct one theory which contains the initial theory and all corresponding T-dual ones. 
We have succeeded to realize such program in the bosonic case, for both constant and weakly curved background. In Refs. [35,36] we doubled all bosonic coordinates and obtain the theory which contains the initial and all corresponding Tdual theories. In such theory T-dualization along an arbitrary set of coordinates $x^{a}$ is equivalent to replacement of these coordinates with the corresponding T-dual ones, $y_{a}$. Therefore, T-duality in double space becomes symmetry transformation with respect to permutation group.

Performing T-duality in supersymmetric case generates new problems. In the present paper we are going to extend such an approach to the type II theories. In fact, doubling all bosonic coordinates we have unified types IIA, IIB as well as type $\mathrm{II}^{\star}$ [37] (obtained by T-dualization along time-like direction) theories. We expect that such a program could be a step toward better understanding M-theory.

In the present article we apply the approach of Refs. [35, $36]$ in the cases of complete (along all bosonic coordinates) and partial (subset of the bosonic coordinates) T-dualization of the type II superstring theory [1-3]. We use ghost free type II superstring theory in pure spinor formulation $[33,38-$ $44]$ in the approximation of constant background fields and up to the quadratic terms. This action is obtained from the general type II superstring action [45] which is given in the form of an expansion in powers of fermionic coordinates $\theta^{\alpha}$ and $\bar{\theta}^{\alpha}$. In the first step of our consideration we will limit our analysis to the basic term of the action neglecting $\theta^{\alpha}$ and $\bar{\theta}^{\alpha}$ dependent terms. Later, in the discussion of proper fermionic variables, using an iterative procedure [45], we take into consideration higher power terms and restore the supersymmetric invariants $\Pi_{ \pm}^{\mu}, d_{\alpha}$ and $\bar{d}_{\alpha}$ as variables in the theory.

Rewriting the T-dual transformation laws in terms of the double space coordinates $Z^{M}$ we introduce the generalized metric $\mathcal{H}_{M N}$ and the generalized current $J_{ \pm M}$. The permutation matrix $\left(\mathcal{T}^{a}\right)^{M}{ }_{N}$ exchanges the places of $x^{a}$ and $y_{a}$, where the index $a$ marks the directions along which we make T-dualization. The basic request is that T-dual double space coordinates, ${ }_{a} Z^{M}=\left(\mathcal{T}^{a}\right)^{M}{ }_{N} Z^{N}$, satisfy the transformation law of the same form as initial coordinates, $Z^{M}$. It produces the expressions for the T-dual generalized metric, ${ }_{a} \mathcal{H}_{M N}=\left(\mathcal{T}^{a} \mathcal{H} \mathcal{T}^{a}\right)_{M N}$, and the T-dual current, ${ }_{a} J_{ \pm M}=\left(\mathcal{T}^{a} J_{ \pm}\right)_{M}$. This is equivalent to the requirement that transformations of the coordinates and background fields, $Z^{M} \rightarrow{ }_{a} Z^{M}, \mathcal{H}_{M N} \rightarrow{ }_{a} \mathcal{H}_{M N}$ and $J_{ \pm M} \rightarrow{ }_{a} J_{ \pm M}$, are symmetry transformations of the double space action. From transformation of the generalized metric we obtain T-dual NS-NS background fields and from transformation of the current we obtain T-dual NS-R fields.

The supersymmetry case includes the new features in both the Buscher and the double space T-duality approaches. In the bosonic case the left and right world-sheet chiralities have different T-duality transformations. It implies that in T- dual theory two fermionic coordinates, $\theta^{\alpha}$ and $\bar{\theta}^{\alpha}$, and corresponding canonically conjugated momenta, $\pi_{\alpha}$ and $\bar{\pi}_{\alpha}$ (with different world-sheet chiralities), have different supersymmetry transformations. As shown in $[46,47]$ it is possible to make a supersymmetry transformation in T-dual theory unique if we change one world-sheet chirality sector. Therefore, compatibility between supersymmetry and T-duality can be achieved by action on the bar variables with the operator ${ }_{a} \Omega, \bullet^{\bullet} \bar{\pi}_{\alpha}={ }_{a} \Omega_{\alpha} \beta{ }_{a} \bar{\pi}_{\beta}$. As a consequence of the relation $\Gamma^{11}{ }_{a} \Omega=(-1)^{d}{ }_{a} \Omega \Gamma^{11}$ it follows that such transformations for odd $d$ change space-time chiralities of the bar spinors. In such a way the operator ${ }_{a} \Omega$ for odd $d$ maps type IIA/B to type IIB/A theory. Here $d$ denotes the number of T-dualized directions.

There is one difference compared with the bosonic string case $[35,36]$ where all results from the Buscher procedure were reproduced. In the T-dual transformation laws of type II superstring theory the $\mathrm{R}-\mathrm{R}$ field strength $F^{\alpha \beta}$ does not appear. The reason is that $\mathrm{R}-\mathrm{R}$ field strength couples only with the fermionic degrees of freedom, which are not dualized. This is in analogy with the term $\partial_{+} x^{i} \Pi_{+i j} \partial_{-} x^{j}$ in the bosonic case, where background field $\Pi_{+i j}$ couples only with coordinates $x^{i}$, which are undualized [27-29]. To reproduce the Buscher form of the T-dual R-R field strength we should make some additional assumptions.

There is an appendix, which contains the block-wise expressions for the tensors used in this article and useful relations.

\section{Buscher T-dualization of type II superstring theory}

In this section we will consider type II superstring action in pure spinor formulation $[38,43,44]$ in the approximation of constant background fields and up to the quadratic terms. Then we will give the overview of the results obtained by Buscher T-dualization procedure [9, 10,46,47].

\subsection{Type II superstring in pure spinor formulation}

The sigma model action for the type II superstring of Ref. [45] is of the form

$S=\int_{\Sigma} \mathrm{d}^{2} \xi\left(X^{T}\right)^{M} A_{M N} \bar{X}^{N}+S_{\lambda}+S_{\bar{\lambda}}$,

where the vectors $X^{M}$ and $\bar{X}^{N}$ are left and right chiral supersymmetric variables,

$X^{M}=\left(\begin{array}{c}\partial_{+} \theta^{\alpha} \\ \Pi_{+}^{\mu} \\ d_{\alpha} \\ \frac{1}{2} N_{+}^{\mu \nu}\end{array}\right), \quad \bar{X}^{N}=\left(\begin{array}{c}\partial_{-} \bar{\theta}^{\alpha} \\ \Pi_{-}^{\mu} \\ \bar{d}_{\alpha}^{\alpha} \\ \frac{1}{2} \bar{N}_{-}^{\mu \nu}\end{array}\right)$, 
of which the components are defined as

$$
\begin{aligned}
\Pi_{+}^{\mu} & =\partial_{+} x^{\mu}+\frac{1}{2} \theta^{\alpha}\left(\Gamma^{\mu}\right)_{\alpha \beta} \partial_{+} \theta^{\beta} \\
\Pi_{-}^{\mu} & =\partial_{-} x^{\mu}+\frac{1}{2} \bar{\theta}^{\alpha}\left(\Gamma^{\mu}\right)_{\alpha \beta} \partial_{-} \bar{\theta}^{\beta} \\
d_{\alpha} & =\pi_{\alpha}-\frac{1}{2}\left(\Gamma_{\mu} \theta\right)_{\alpha}\left[\partial_{+} x^{\mu}+\frac{1}{4}\left(\theta \Gamma_{\mu} \partial_{+} \theta\right)\right] \\
\bar{d}_{\alpha} & =\bar{\pi}_{\alpha}-\frac{1}{2}\left(\Gamma_{\mu} \bar{\theta}\right)_{\alpha}\left[\partial_{-} x^{\mu}+\frac{1}{4}\left(\bar{\theta} \Gamma_{\mu} \partial_{-} \bar{\theta}\right)\right], \\
N_{+}^{\mu \nu} & =\frac{1}{2} w_{\alpha}\left(\Gamma^{[\mu \nu]}\right)^{\alpha}{ }_{\beta} \lambda^{\beta}, \quad \bar{N}_{-}^{\mu \nu}=\frac{1}{2} \bar{w}_{\alpha}\left(\Gamma^{[\mu \nu]}\right)^{\alpha}{ }_{\beta} \bar{\lambda}^{\beta} .
\end{aligned}
$$

Inserting the supermatrix $A_{M N}$

$$
A_{M N}=\left(\begin{array}{cccc}
A_{\alpha \beta} & A_{\alpha \nu} & E_{\alpha}^{\beta} & \Omega_{\alpha, \mu \nu} \\
A_{\mu \beta} & A_{\mu \nu} & \bar{E}_{\mu}^{\beta} & \Omega_{\mu, v \rho} \\
E_{\beta}^{\alpha} & E_{\nu}^{\alpha} & \mathrm{P}^{\alpha \beta} & C^{\alpha}{ }_{\mu \nu} \\
\Omega_{\mu \nu, \beta} & \Omega_{\mu \nu, \rho} & \bar{C}_{\mu \nu} \beta & S_{\mu \nu, \rho \sigma}
\end{array}\right),
$$

in (2.1), the action gets the expanded form [45]

$$
\begin{aligned}
S= & \int \mathrm{d}^{2} \xi\left[\partial_{+} \theta^{\alpha} A_{\alpha \beta} \partial_{-} \bar{\theta}^{\beta}+\partial_{+} \theta^{\alpha} A_{\alpha \mu} \Pi_{-}^{\mu}+\Pi_{+}^{\mu} A_{\mu \alpha} \partial_{-} \bar{\theta}^{\alpha}\right. \\
& +\Pi_{+}^{\mu} A_{\mu \nu} \Pi_{-}^{v}+d_{\alpha} E^{\alpha}{ }_{\beta} \partial_{-} \bar{\theta}^{\beta}+d_{\alpha} E^{\alpha}{ }_{\mu} \Pi_{-}^{\mu} \\
& +\partial_{+} \theta^{\alpha} E_{\alpha} \beta \bar{d}_{\beta}+\Pi_{+}^{\mu} E_{\mu} \beta \bar{d}_{\beta}+d_{\alpha} \mathrm{P}^{\alpha \beta} \bar{d}_{\beta} \\
& +\frac{1}{2} N_{+}^{\mu \nu} \Omega_{\mu \nu, \beta} \partial_{-} \bar{\theta}^{\beta}+\frac{1}{2} N_{+}^{\mu \nu} \Omega_{\mu \nu, \rho} \Pi_{-}^{\rho} \\
& +\frac{1}{2} \partial_{+} \theta^{\alpha} \Omega_{\alpha, \mu \nu} \bar{N}_{-}^{\mu \nu}+\frac{1}{2} \Pi_{+}^{\mu} \Omega_{\mu, \nu \rho} \bar{N}_{-}^{v \rho} \\
& +\frac{1}{2} N_{+}^{\mu \nu} \bar{C}_{\mu \nu} \beta \bar{d}_{\beta}+\frac{1}{2} d_{\alpha} C^{\alpha}{ }_{\mu \nu} \bar{N}_{-}^{\mu \nu} \\
& \left.+\frac{1}{4} N_{+}^{\mu \nu} S_{\mu \nu, \rho \sigma} \bar{N}_{-}^{\rho \sigma}\right]+S_{\lambda}+S_{\bar{\lambda} .}
\end{aligned}
$$

The world sheet $\Sigma$ is parameterized by $\xi^{m}=\left(\xi^{0}=\tau, \xi^{1}=\right.$ $\sigma)$ and $\partial_{ \pm}=\partial_{\tau} \pm \partial_{\sigma}$. Superspace is spanned by bosonic coordinates, $x^{\mu}(\mu=0,1,2, \ldots, 9)$, and fermionic ones, $\theta^{\alpha}$ and $\bar{\theta}^{\alpha}(\alpha=1,2, \ldots, 16)$. The variables $\pi_{\alpha}$ and $\bar{\pi}_{\alpha}$ are canonically conjugated momenta to $\theta^{\alpha}$ and $\bar{\theta}^{\alpha}$, respectively. The actions for pure spinors, $S_{\lambda}$ and $S_{\bar{\lambda}}$, are free field actions

$S_{\lambda}=\int \mathrm{d}^{2} \xi w_{\alpha} \partial_{-} \lambda^{\alpha}, \quad S_{\bar{\lambda}}=\int \mathrm{d}^{2} \xi \bar{w}_{\alpha} \partial_{+} \bar{\lambda}^{\alpha}$,

where $\lambda^{\alpha}$ and $\bar{\lambda}^{\alpha}$ are pure spinors and $w_{\alpha}$ and $\bar{w}_{\alpha}$ are their canonically conjugated momenta, respectively. The pure spinors satisfy the so-called pure spinor constraints,

$\lambda^{\alpha}\left(\Gamma^{\mu}\right)_{\alpha \beta} \lambda^{\beta}=\bar{\lambda}^{\alpha}\left(\Gamma^{\mu}\right)_{\alpha \beta} \bar{\lambda}^{\beta}=0$.

The matrix $A_{M N}$ containing type II superfields generally depends on $x^{\mu}, \theta^{\alpha}$ and $\bar{\theta}^{\alpha}$. The superfields $A_{\mu \nu}, \bar{E}_{\mu}{ }^{\alpha}, E^{\alpha}{ }_{\mu}$ and $\mathrm{P}^{\alpha \beta}$ are physical superfields, because their first components are supergravity fields. The fields in the first column and first row are auxiliary superfields because they can be expressed in terms of the physical ones [45]. The remaining ones, $\Omega_{\mu, \nu \rho}\left(\Omega_{\mu \nu, \rho}\right), C^{\alpha}{ }_{\mu \nu}\left(\bar{C}_{\mu \nu}{ }^{\alpha}\right)$ and $S_{\mu \nu, \rho \sigma}$, are the curvatures (field strengths) for the physical superfields.

The action from which we start (2.7) could be considered as an expansion in powers of $\theta^{\alpha}$ and $\bar{\theta}^{\alpha}$. In the iterative procedure presented in [45] it has been shown that each component in the expansion can be obtained from the previous one. Therefore, for practical reasons (computational simplicity), in the first step we limit our considerations to the basic component i.e. we neglect all terms in the action containing $\theta^{\alpha}$ and $\bar{\theta}^{\alpha}$. As a consequence the $\theta^{\alpha}$ and $\bar{\theta}^{\alpha}$ terms disappear from $\Pi_{ \pm}^{\mu}, d_{\alpha}$ and $\bar{d}_{\alpha}$ and in the solutions for the physical superfields just $x$-dependent supergravity fields survive. Therefore we lose explicit supersymmetry in such approximation. Later, when we discuss proper fermionic variables, we would go further in the expansion and take higher power terms, which means that supersymmetric invariants, $\Pi_{ \pm}^{\mu}, d_{\alpha}$ and $\bar{d}_{\alpha}$, would play the roles of $\partial_{ \pm} x^{\mu}, \pi_{\alpha}$ and $\bar{\pi}_{\alpha}$, respectively.

We are going to perform T-dualization along some subset of bosonic coordinates $x^{a}$. Therefore, we will assume that these directions are Killing vectors. Since $\partial_{ \pm} x^{a}$ appears in $\Pi_{ \pm}^{\mu}, d_{\alpha}$ and $\bar{d}_{\alpha}$, it essentially means that corresponding superfields $\left(A_{a b}, \bar{E}_{a}{ }^{\alpha}, E^{\alpha}{ }_{a}, \mathrm{P}^{\alpha \beta}\right)$ should not depend on $x^{a}$. This assumption regarding Killing spinors could be extended on all space-time directions $x^{\mu}$, which effectively means, in the first step, that physical superfields are constant. All auxiliary superfields can be expressed in terms of space-time derivatives of physical supergravity fields [45]. Then, in the first step, the auxiliary superfields are zero, because all physical superfields are constant. On the other hand, having constant physical superfields means that their field strengths, $\Omega_{\mu, \nu \rho}\left(\Omega_{\mu \nu, \rho}\right), C_{\mu \nu}^{\alpha}\left(\bar{C}_{\mu \nu}{ }^{\alpha}\right)$ and $S_{\mu \nu, \rho \sigma}$, are zero. In this way, in the first step, we eliminated from the action terms containing variables $N_{+}^{\mu \nu}$ and $\bar{N}_{-}^{\mu \nu}(2.5)$.

This choice of background fields should be discussed from the viewpoint of space-time field equations of type II superstring action [48]. Let us pay attention on the space-time field equations for type II superstring given in Appendix B of [48]. Equation (B.7) from this set of equations represents the back-reaction of $\mathrm{P}^{\alpha \beta}$ on the metric $G_{\mu \nu}$. If we take a constant dilaton $\Phi$ and a constant antisymmetric NS-NS field $B_{\mu \nu}$ we obtain

$R_{\mu \nu}-\frac{1}{2} G_{\mu \nu} R \sim\left(\mathrm{P}^{\alpha \beta}\right)_{\mu \nu}^{2}$.

If we choose the background field $\mathrm{P}^{\alpha \beta}$ to be constant, in general, we will have a constant Ricci tensor, which means that the metric tensor is a quadratic function of the space-time coordinates i.e. there is back-reaction of $\mathrm{R}-\mathrm{R}$ field strength on the metric tensor. If one wants to cancel non-quadratic terms originating from back-reaction, additional conditions 
must be imposed on the R-R field strength-the $A d S_{5} \times S_{5}$ coset geometry or self-duality condition (see Ref. [38]).

Taking into account the above analysis and arguments, our approximation can be realized in the following way:

$\Pi_{ \pm}^{\mu} \rightarrow \partial_{ \pm} x^{\mu}, \quad d_{\alpha} \rightarrow \pi_{\alpha}, \quad \bar{d}_{\alpha} \rightarrow \bar{\pi}_{\alpha}$

and the physical superfields take the form

$$
\begin{gathered}
A_{\mu \nu}=\kappa\left(\frac{1}{2} G_{\mu \nu}+B_{\mu \nu}\right), \quad E_{\nu}^{\alpha}=-\Psi_{\nu}^{\alpha}, \quad \bar{E}_{\mu}^{\alpha}=\bar{\Psi}_{\mu}^{\alpha}, \\
\mathrm{P}^{\alpha \beta}=\frac{2}{\kappa} P^{\alpha \beta}=\frac{2}{\kappa} e^{\frac{\Phi}{2}} F^{\alpha \beta},
\end{gathered}
$$

where $G_{\mu \nu}$ is the metric tensor and $B_{\mu \nu}$ is the antisymmetric NS-NS background field. Consequently, the full action $S$ is

$$
\begin{aligned}
S= & \kappa \int_{\Sigma} \mathrm{d}^{2} \xi\left[\partial_{+} x^{\mu} \Pi_{+\mu \nu} \partial_{-} x^{\nu}+\frac{1}{4 \pi \kappa} \Phi R^{(2)}\right] \\
& +\int_{\Sigma} \mathrm{d}^{2} \xi\left[-\pi_{\alpha} \partial_{-}\left(\theta^{\alpha}+\Psi_{\mu}^{\alpha} x^{\mu}\right)+\partial_{+}\left(\bar{\theta}^{\alpha}+\bar{\Psi}_{\mu}^{\alpha} x^{\mu}\right) \bar{\pi}_{\alpha}\right. \\
& \left.+\frac{2}{\kappa} \pi_{\alpha} P^{\alpha \beta} \bar{\pi}_{\beta}\right],
\end{aligned}
$$

where

$\Pi_{ \pm \mu \nu}=B_{\mu \nu} \pm \frac{1}{2} G_{\mu \nu}$.

The actions $S_{\lambda}$ and $S_{\bar{\lambda}}$ are decoupled from the rest and can be neglected in the further analysis. The action, in its final form, is ghost independent.

The NS-NS sector of the theory described by (2.13) contains the gravitational $G_{\mu \nu}$, the antisymmetric Kalb-Ramond field $B_{\mu \nu}$ and the dilaton field $\Phi$. In the NS-R sector there are two gravitino fields, $\Psi_{\mu}^{\alpha}$ and $\bar{\Psi}_{\mu}^{\alpha}$, which are Majorana-Weyl spinors of the opposite chirality in type IIA and of the same chirality in type IIB theory. The field $F^{\alpha \beta}$ is the R-R field strength and can be expressed in terms of the antisymmetric tensors $F_{(k)}[9,49-51]$

$F^{\alpha \beta}=\sum_{k=0}^{D} \frac{1}{k !} F_{(k)} \Gamma_{(k)}^{\alpha \beta}, \quad\left[\Gamma_{(k)}^{\alpha \beta}=\left(\Gamma^{\left[\mu_{1} \cdots \mu_{k}\right]}\right)^{\alpha \beta}\right]$

where

$\Gamma^{\left[\mu_{1} \mu_{2} \cdots \mu_{k}\right]} \equiv \Gamma^{\left[\mu_{1}\right.} \Gamma^{\mu_{2}} \cdots \Gamma^{\left.\mu_{k}\right]}$

is the completely antisymmetrized product of gamma matrices. The bispinor $F^{\alpha \beta}$ satisfies the chirality condition, $\Gamma^{11} F= \pm F \Gamma^{11}$, where $\Gamma^{11}$ is a product of gamma matrices in $D=10$ dimensional space-time and the sign + corresponds to type IIA, while the sign - corresponds to type IIB superstring theory. Consequently, type IIA theory contains only even rank tensors $F_{(k)}$, while type IIB contains only odd rank tensors. Because of the duality relation, the independent tensors are $F_{(0)}, F_{(2)}$ and $F_{(4)}$ for type IIA, while $F_{(1)}, F_{(3)}$ and the self-dual part of $F_{(5)}$ for type IIB superstring theory. Using the mass-shell condition (massless Dirac equation for $F^{\alpha \beta}$ ) these tensors can be solved in terms of the potentials $F_{(k)}=d A_{(k-1)}$. The factor $e^{\frac{\Phi}{2}}$ is in accordance with the conventions adopted from [52].

\subsection{T-dualization along arbitrary number of coordinates}

Let us start with the action (2.13) and apply the standard Tdualization procedure $[4,5,19,20]$. It means that we localize the shift symmetry for some coordinates $x^{a}$. We substitute the ordinary derivatives with covariant ones, introducing gauge fields $v_{\alpha}^{a}$. Then we add the term $\frac{1}{2} y_{a} F_{+-}^{a}$ to the Lagrangian in order to force the field strength $F_{+-}^{a}$ to vanish and preserve equivalence between original and T-dual theories. Finally, we fix the gauge $x^{a}=0$ and obtain

$$
\begin{aligned}
& S_{\mathrm{fix}}\left(v_{ \pm}^{a}, x^{i}, \theta^{\alpha}, \bar{\theta}^{\alpha}, \pi_{\alpha}, \bar{\pi}_{\alpha}\right) \\
& =\int_{\Sigma} \mathrm{d}^{2} \xi\left[\kappa v_{+}^{a} \Pi_{+a b} v_{-}^{b}+\kappa v_{+}^{a} \Pi_{+a j} \partial_{-} x^{j}+\kappa \partial_{+} x^{i} \Pi_{+i b} v_{-}^{b}\right. \\
& \quad+\kappa \partial_{+} x^{i} \Pi_{+i j} \partial_{-} x^{j}+\frac{1}{4 \pi} \Phi R^{(2)}-\pi_{\alpha} \Psi_{b}^{\alpha} v_{-}^{b} \\
& \quad+v_{+}^{a} \bar{\Psi}_{a}^{\alpha} \bar{\pi}_{\alpha}-\pi_{\alpha} \partial_{-}\left(\theta^{\alpha}+\Psi_{i}^{\alpha} x^{i}\right)+\partial_{+}\left(\bar{\theta}^{\alpha}+\bar{\Psi}_{i}^{\alpha} x^{i}\right) \bar{\pi}_{\alpha} \\
& \left.\quad+\frac{1}{2 \kappa} e^{\frac{\Phi}{2}} \pi_{\alpha} F^{\alpha \beta} \bar{\pi}_{\beta}+\frac{\kappa}{2}\left(v_{+}^{a} \partial_{-} y_{a}-v_{-}^{a} \partial_{+} y_{a}\right)\right] \cdot
\end{aligned}
$$

Varying the gauge fixed action with respect to the Lagrange multipliers $y_{a}$ we get the solution for the gauge fields in the form

$v_{ \pm}^{a}=\partial_{ \pm} x^{a}$

while varying with respect to the gauge fields $v_{ \pm}^{a}$ we have

$v_{ \pm}^{a}=-2 \kappa \hat{\theta}_{ \pm}^{a b} \Pi_{\mp b i} \partial_{ \pm} x^{i}-\kappa \hat{\theta}_{ \pm}^{a b} \partial_{ \pm} y_{b} \pm 2 \hat{\theta}_{ \pm}^{a b} \Psi_{ \pm b}^{\alpha} \pi_{ \pm \alpha}$.

Substituting $v_{ \pm}^{a}$ in (2.17) we find

$$
\begin{aligned}
& S_{\mathrm{fix}}\left(y_{a}, x^{i}, \theta^{\alpha}, \bar{\theta}^{\alpha}, \pi_{\alpha}, \bar{\pi}_{\alpha}\right) \\
& =\int_{\Sigma} \mathrm{d}^{2} \xi\left[\frac{\kappa^{2}}{2} \partial_{+} y_{a} \hat{\theta}_{-}^{a b} \partial_{-} y_{b}+\kappa^{2} \partial_{+} y_{a} \hat{\theta}_{-}^{a b} \Pi_{+b j} \partial_{-} x^{j}\right. \\
& \quad-\kappa^{2} \partial_{+} x^{i} \Pi_{+i a} \hat{\theta}_{-}^{a b} \partial_{-} y_{b}+\frac{1}{4 \pi} \Phi R^{(2)} \\
& \quad+\kappa \partial_{+} x^{i}\left(\Pi_{+i j}-2 \kappa \Pi_{+i a} \hat{\theta}_{-}^{a b} \Pi_{+b j}\right) \partial_{-} x^{j} \\
& \quad-\pi_{\alpha} \partial_{-}\left(\theta^{\alpha}+\Psi_{i}^{\alpha} x^{i}-2 \Psi_{a}^{\alpha} \hat{\theta}_{-}^{a b} \Pi_{+b j} x^{j}-\Psi_{a}^{\alpha} \hat{\theta}_{-}^{a b} y_{b}\right) \\
& \quad+\partial_{+}\left(\bar{\theta}^{\alpha}+\bar{\Psi}_{i}^{\alpha} x^{i}+2 \bar{\Psi}_{a}^{\alpha} \hat{\theta}_{+}^{a b} \Pi_{-b j} x^{j}+\bar{\Psi}_{a}^{\alpha} \hat{\theta}_{+}^{a b} y_{b}\right) \bar{\pi}_{\alpha} \\
& \left.\quad+2 \pi_{\alpha} \Psi_{a}^{\alpha} \hat{\theta}_{-}^{a b} \bar{\Psi}_{b}^{\beta} \bar{\pi}_{\beta}+\frac{1}{2 \kappa} e^{\frac{\Phi}{2}} \pi_{\alpha} F^{\alpha \beta} \bar{\pi}_{\beta}\right] .
\end{aligned}
$$

Before we read the T-dual background fields, we must express this action in terms of the appropriate spinor coordinates, which we will discuss in the next subsections.

Combining two solutions for the gauge fields (2.18) and (2.19) we obtain the transformation law between initial $x^{a}$ 
and T-dual coordinates $y_{a}$,

$\partial_{ \pm} x^{a} \cong-2 \kappa \hat{\theta}_{ \pm}^{a b} \Pi_{\mp b i} \partial_{ \pm} x^{i}-\kappa \hat{\theta}_{ \pm}^{a b}\left(\partial_{ \pm} y_{b}-J_{ \pm b}\right)$.

Its inverse is the solution of the last equation in terms of $y_{a}$

$\partial_{ \pm} y_{a} \cong-2 \Pi_{\mp a b} \partial_{ \pm} x^{b}-2 \Pi_{\mp a i} \partial_{ \pm} x^{i}+J_{ \pm a}$,

where we use $\cong$ to emphasize that these are T-duality relations. Here we introduced the current $J_{ \pm \mu}$ in the form

$J_{ \pm \mu}= \pm \frac{2}{\kappa} \Psi_{ \pm \mu}^{\alpha} \pi_{ \pm \alpha}$

where

$\Psi_{+\mu}^{\alpha} \equiv \Psi_{\mu}^{\alpha}, \quad \Psi_{-\mu}^{\alpha} \equiv \bar{\Psi}_{\mu}^{\alpha}, \quad \pi_{+\alpha} \equiv \pi_{\alpha}, \quad \pi_{-\alpha} \equiv \bar{\pi}_{\alpha}$,

and the expression $\hat{\theta}_{ \pm}^{a b}$ is defined in (A.9).

\subsection{Relation between left and right chirality in T-dual theory}

One can see from (2.21) and (2.22) that the left and right chiralities transform differently in T-dual theory. As a consequence, in T-dual theory we will have two types of vielbeins, two types of $\Gamma$-matrices, two types of spin connections and two types of supersymmetry transformations. We want to have a single geometry in T-dual theory. Therefore, we will show that all these different representations of the same variables can be connected by Lorentz transformations [46,47].

\subsubsection{Two sets of vielbeins in T-dual theory}

The T-dual transformations of the coordinates (2.22) can be put in the form

$$
\left(\begin{array}{c}
\partial_{ \pm} y_{a} \\
\partial_{ \pm} x^{i}
\end{array}\right)=\left(\begin{array}{cc}
-2 \Pi_{\mp a b} & -2 \Pi_{\mp a j} \\
0 & \delta_{j}^{i}
\end{array}\right)\left(\begin{array}{l}
\partial_{ \pm} x^{b} \\
\partial_{ \pm} x^{j}
\end{array}\right)+\left(\begin{array}{c}
J_{ \pm a} \\
0
\end{array}\right),
$$

which can be rewritten as

$$
\begin{gathered}
\partial_{+}\left({ }_{a} X\right)_{\hat{\mu}}=\left(\bar{Q}^{-1 T}\right)_{\hat{\mu} \nu} \partial_{+} x^{\nu}+J_{+\hat{\mu}}, \\
\partial_{-}\left({ }_{a} X\right)_{\hat{\mu}}=\left(Q^{-1 T}\right)_{\hat{\mu} \nu} \partial_{-} x^{\nu}+J_{-\hat{\mu}},
\end{gathered}
$$

where we introduced the T-dual variables ${ }_{a} X_{\hat{\mu}}=\left\{y_{a}, x^{i}\right\}$. Here and further on the left subscript $a$ denotes the Tdualization along $x^{a}$ directions. For coordinates which contain both $x^{i}$ and $y_{a}$ we will use "hat" indices $\hat{\mu}, \hat{v}$. The matrices

$$
Q^{\hat{\mu} \nu}=\left(\begin{array}{cc}
\kappa \hat{\theta}_{+}^{a b} & 0 \\
-2 \kappa \Pi_{-i c} \hat{\theta}_{+}^{c b} & \delta_{j}^{i}
\end{array}\right), \quad \bar{Q}^{\hat{\mu} \nu}=\left(\begin{array}{cc}
\kappa \hat{\theta}_{-}^{a b} & 0 \\
-2 \kappa \Pi_{+i c} \hat{\theta}_{-}^{c b} & \delta_{j}^{i}
\end{array}\right),
$$

and theirs inverse

$Q_{\mu \hat{v}}^{-1}=\left(\begin{array}{cc}2 \Pi_{-a b} & 0 \\ 2 \Pi_{-i b} & \delta_{i}^{j}\end{array}\right), \quad \bar{Q}_{\mu \hat{\nu}}^{-1}=\left(\begin{array}{cc}2 \Pi_{+a b} & 0 \\ 2 \Pi_{+i b} & \delta_{i}^{j}\end{array}\right)$,

perform T-dualization for the vector indices.

Note that different chiralities transform with different matrices $Q^{\hat{\mu} v}$ and $\bar{Q}^{\hat{\mu} v}$. Therefore, there are two types of T-dual vielbeins

${ }_{a} e^{\underline{a} \hat{\mu}}=e^{\underline{a}}{ }_{\nu}\left(Q^{T}\right)^{\nu \hat{\mu}}, \quad{ }_{a} e^{\underline{a} \hat{\mu}}=e^{\underline{a}}{ }_{\nu}\left(\bar{Q}^{T}\right)^{\nu \hat{\mu}}$,

with the same T-dual metric

$$
\begin{aligned}
{ }_{a} G^{\hat{\mu} \hat{\nu}} & \equiv\left({ }_{a} e^{T} \eta_{a} e\right)^{\hat{\mu} \hat{\nu}}=\left(Q G Q^{T}\right)^{\hat{\mu} \hat{\nu}}={ }_{a} \bar{G}^{\hat{\mu} \hat{\nu}} \\
& \equiv\left({ }_{a} e^{T} \eta_{a} \bar{e}\right)^{\hat{\mu} \hat{v}}=\left(\bar{Q} G \bar{Q}^{T}\right)^{\bar{\mu} \hat{\nu}} .
\end{aligned}
$$

The Lorentz indices are underlined (denoted by $\underline{a}, \underline{b}$ ).

The two T-dual vielbeins are equivalent because they are related by the particular local Lorentz transformation

${ }_{a} e^{\underline{a} \hat{\mu}}=\Lambda^{\underline{a}} \underline{b}_{a} e^{\underline{b} \hat{\mu}}, \quad \Lambda^{\underline{a}} \underline{b}=e^{\underline{a}} \mu\left(Q^{-1} \bar{Q}\right)^{T \mu}{ }_{\nu}\left(e^{-1}\right)^{v}{ }_{\underline{b}}$.

From (2.27) and (2.28) we have

$\left(Q^{-1} \bar{Q}\right)^{T \mu_{\nu}}=\left(\begin{array}{cc}\delta^{a}{ }_{b}+2 \kappa \hat{\theta}_{+}^{a c} G_{c b} & 2 \kappa \hat{\theta}_{+}^{a c} G_{c j} \\ 0 & \delta_{j}^{i}\end{array}\right)$,

which produces

$\Lambda^{\underline{a}_{\underline{b}}}=\delta^{\underline{a}} \underline{\underline{b}}_{\underline{b}}-2 \omega^{\underline{a}} \underline{\underline{b}}_{\underline{b}}, \quad \omega^{\underline{a}} \underline{\underline{b}}_{\underline{b}}=-\kappa e^{\underline{a}}{ }_{a} \hat{\theta}_{+}^{a b}\left(e^{T}\right)_{b^{\underline{c}}} \eta_{\underline{c b}}$.

It satisfies definition of Lorentz transformations

$\Lambda^{T} \eta \Lambda=\eta \quad \Longrightarrow \quad \operatorname{det} \Lambda^{\underline{a}} \underline{b}_{b}= \pm 1$.

After careful calculations we have det $\Lambda^{a}{ }^{a} \underline{b}=(-1)^{d}$, where $d$ is the number of dimensions along which we perform Tduality.

\subsubsection{Two sets of $\Gamma$-matrices in T-dual theory}

Because in T-dual theory there are two vielbeins, there must also be two sets of $\Gamma$-matrices in curved space

$$
\begin{gathered}
{ }_{a} \Gamma_{\hat{\mu}}=\left({ }_{a} e^{-1}\right)_{\hat{\mu} \underline{a}} \Gamma^{\underline{a}}=\left({ }_{a} e^{-1} \Gamma\right)_{\hat{\mu}}, \\
{ }_{a} \bar{\Gamma}_{\hat{\mu}}=\left({ }_{a} \bar{e}^{-1}\right)_{\hat{\mu} \underline{a}} \Gamma^{\underline{a}}=\left({ }_{a} \bar{e}^{-1} \Gamma\right)_{\hat{\mu}} .
\end{gathered}
$$

They are related by the expression

${ }_{a} \bar{\Gamma}_{\hat{\mu}}={ }_{a} \Omega^{-1}{ }_{a} \Gamma_{\hat{\mu}}{ }_{a} \Omega$,

where ${ }_{a} \Omega$ is a spinorial representation of the Lorentz transformation

${ }_{a} \Omega^{-1} \Gamma^{\underline{a}}{ }_{a} \Omega=\left(\Lambda^{-1}\right)^{\underline{a}} \underline{b} \Gamma^{\underline{b}}$. 


\subsubsection{Two sets of spin connections in T-dual theory}

The spin connection can be expressed in terms of vielbeins as

$\omega_{\mu} \underline{a b}=\frac{1}{2}\left(e^{\nu \underline{a}} c_{\mu}^{\underline{\mu} \nu}-e^{\nu \underline{b}} c_{\mu}^{\underline{\mu} \nu}\right)-\frac{1}{2} e^{\rho \underline{a}} e^{\sigma \underline{b}} c_{\underline{c} \rho \sigma} e^{\underline{c}} \mu$,

where

$c^{\frac{a}{\mu} \nu}=\partial_{\mu} e^{\underline{a}}{ }_{\nu}-\partial_{\nu} e^{\underline{a}} \mu$.

Therefore, in T-dual theory there are two spin connections, defined in terms of two vielbeins. As a consequence of (2.31) they are related as

${ }_{a} \bar{\omega} \hat{\mu} \underline{a}_{\underline{b}}=\Lambda^{\underline{a}} \underline{c}_{a} \omega^{\hat{\mu} \underline{c}_{\underline{d}}}\left(\Lambda^{-1}\right) \underline{d}_{\underline{b}}+\Lambda^{\underline{a}} \underline{c}_{\underline{c}} \partial^{\hat{\mu}}\left(\Lambda^{-1}\right) \underline{c}_{\underline{b}}$.

It is useful to introduce the spin connection in the form

$\omega_{\mu}=\omega_{\mu \underline{a b}} \Gamma \underline{a b}$,

where

$\Gamma^{\underline{a b}}=\Gamma^{\underline{a}} \Gamma^{\underline{b}}-\Gamma^{\underline{b}} \Gamma^{\underline{a}}$.

Then from (2.37) for ${ }_{a} \Omega=$ const we obtain

${ }_{a} \bar{\omega}^{\hat{\mu}}={ }_{a} \Omega^{-1}{ }_{a} \omega^{\hat{\mu}}{ }_{a} \Omega$.

\subsubsection{Single form of supersymmetry invariants in T-dual theory and new spinor coordinates}

So far we used the action from Ref. [45] which is an expansion in powers of $\theta^{\alpha}$ and $\bar{\theta}^{\alpha}$. We performed the procedure of bosonic T-dualization using the first term in the expansion i.e. $\theta^{\alpha}$ and $\bar{\theta}^{\alpha}$ independent part of the action. Consequently, the supersymmetric invariants, $\Pi_{ \pm}^{\mu}, d_{\alpha}$ and $\bar{d}_{\alpha}$, in that approximation became $\partial_{ \pm} x^{\mu}, \pi_{\alpha}$ and $\bar{\pi}_{\alpha}$. But if we would take higher power terms into consideration, then these invariants would appear again in the theory. Consequently, we can use these invariants to find proper spinor variables.

From the compatibility between supersymmetry and Tduality we will find appropriate spinor variables changing the bar ones. We are not going to apply such a procedure to background fields which transformation we will find from Tdualization. In Sect. 2.5 we will check that both T-dual gravitinos satisfy a single supersymmetry transformation rule.

Note that according to [38,53-59] fermionic coordinates, $\theta^{\alpha}$ and $\bar{\theta}^{\alpha}$, and their canonically conjugated momenta, $\pi_{\alpha}$ and $\bar{\pi}_{\alpha}$, are parts of the supersymmetry invariant variables,

$d_{\alpha}=\pi_{\alpha}-\frac{1}{2}\left(\Gamma_{\mu} \theta\right)_{\alpha}\left(\partial_{+} x^{\mu}+\frac{1}{4} \theta \Gamma^{\mu} \partial_{+} \theta\right)$

$\bar{d}_{\alpha}=\bar{\pi}_{\alpha}-\frac{1}{2}\left(\Gamma_{\mu} \bar{\theta}\right)_{\alpha}\left(\partial_{-} x^{\mu}+\frac{1}{4} \bar{\theta} \Gamma^{\mu} \partial_{-} \bar{\theta}\right)$.
In T-dual theory, as a consequence of two types of $\Gamma$ matrices, there are two types supersymmetry invariant variables,

$$
\begin{aligned}
& { }_{a} d_{\alpha}={ }_{a} \pi_{\alpha}-\frac{1}{2}\left({ }_{a} \Gamma^{\hat{\mu}}{ }_{a} \theta\right)_{\alpha}\left(\partial_{+}{ }_{a} X_{\hat{\mu}}+\frac{1}{4}{ }_{a} \theta \theta_{a} \Gamma_{\hat{\mu}} \partial_{+}{ }_{a} \theta\right), \\
& { }_{a} \bar{d}_{\alpha}={ }_{a} \bar{\pi}_{\alpha}-\frac{1}{2}\left({ }_{a} \bar{\Gamma}^{\hat{\mu}}{ }_{a} \bar{\theta}\right)_{\alpha}\left(\partial_{-}{ }_{a} X_{\hat{\mu}}+\frac{1}{4} a \bar{\theta}_{a} \bar{\Gamma}_{\hat{\mu}} \partial_{-}{ }_{a} \bar{\theta}\right) .
\end{aligned}
$$

We want the two expressions to have the same $\Gamma$ matrices. Using Eq. (2.36) we can rewrite the bar expressions as

$$
\begin{aligned}
\left({ }_{a} \Omega_{a} \bar{d}\right)_{\alpha}= & \left({ }_{a} \Omega_{a} \bar{\pi}\right)_{\alpha}-\frac{1}{2}\left({ }_{a} \Gamma^{\hat{\mu}}{ }_{a} \Omega_{a} \bar{\theta}\right)_{\alpha}\left(\partial_{-}{ }_{a} X_{\hat{\mu}}\right. \\
& \left.+\frac{1}{4} a \bar{\theta}{ }_{a} \Omega^{-1}{ }_{a} \Gamma_{\hat{\mu}}{ }_{a} \Omega \partial_{-a} \bar{\theta}\right) .
\end{aligned}
$$

Therefore, if we preserve expressions for ${ }_{a} \theta^{\alpha}=\theta^{\alpha}$ and ${ }_{a} \pi_{\alpha}=\pi_{\alpha}$, change bar variables

$\bullet \bar{\theta}^{\alpha} \equiv{ }_{a} \Omega^{\alpha}{ }_{\beta}{ }_{a} \bar{\theta}^{\beta}, \quad \bullet \bar{\pi}_{\alpha} \equiv{ }_{a} \Omega_{\alpha}{ }^{\beta}{ }_{a} \bar{\pi}_{\beta}$,

and take

$\Omega^{2}=1$,

the transformation with bar variables will get the same form as those without bar in ${ }_{a} d_{\alpha}$. Consequently, the T-dual supersymmetric invariant variables ${ }_{a} d_{\alpha}$ and ${ }_{a} \Omega_{\alpha}{ }^{\beta}{ }_{a} \bar{d}_{\beta}$ are expressed in a unique form in terms of the true T-dual spinor variables $\theta^{\alpha}, \pi_{\alpha}, \bullet^{\bullet} \bar{\theta}^{\alpha}$ and ${ }^{\bullet} \bar{\pi}_{\alpha}$,

$$
\begin{aligned}
& { }_{a} d_{\alpha}=d_{\alpha}, \quad \bullet \bar{d}_{\alpha}={ }_{a} \Omega_{\alpha}{ }^{\beta} \bar{d}_{\beta}=\bullet \bar{\pi}_{\alpha}-\frac{1}{2}\left({ }_{a} \Gamma^{\hat{\mu} \bullet \bar{\theta}}\right)_{\alpha} \\
& \quad \times\left(\partial_{-} X_{\hat{\mu}}+\frac{1}{4} \bullet \bar{\theta}{ }_{a} \Gamma_{\hat{\mu}} \partial_{-} \bullet \bar{\theta}\right),
\end{aligned}
$$

if condition (2.49) is satisfied.

\subsubsection{Spinorial representation of the Lorentz transformation}

In order to find expressions for the bar spinors in a T-dual background we should first solve Eq. (2.37) and find the expression for ${ }_{a} \Omega$. We will do it for $B_{\mu \nu} \rightarrow 0$, so that $\hat{\theta}_{+}^{a b} \rightarrow-\frac{1}{\kappa}\left(\widetilde{G}^{-1}\right)^{a b}$, where $\widetilde{G}_{a b}$ is the $a b$ component of $G_{\mu \nu}$. Then from (2.33) it follows that

${ }_{a} \omega^{\underline{a b}} \rightarrow e^{\underline{a}}{ }_{a}\left(\widetilde{G}^{-1}\right)^{a b}\left(e^{T}\right) b^{\underline{b}} \equiv{ }_{a} P^{\underline{a b}}$,

where ${ }_{a} P \underline{a}_{b}$ is some $a$ dependent projector on the $\underline{a b}$ subspace ${ }_{a} P^{a}{ }_{c}{ }_{a} P{ }^{c}{ }_{b}={ }_{a} P{ }^{a} b$. If we introduce the $\Gamma$-matrices in curved space

$\Gamma^{\mu}=\left(e^{-1}\right)^{\mu}{ }_{\underline{a}} \Gamma^{\underline{a}}$,

we can rewrite Eq. (2.37) in the form

${ }_{a} \Omega \Gamma^{\mu}=\left[\Gamma^{\mu}-2\left(\left(e^{-1}\right)^{\mu}{ }_{\underline{a} a} P^{\underline{a}}{ }_{\underline{b}} \Gamma^{\underline{b}}\right]{ }_{a} \Omega\right.$. 
To simplify the derivation from now on we will suppose that the metric tensor is diagonal. Then $\left(e^{-1}\right)^{\mu_{a} a} P_{\underline{\underline{a}}} \underline{\underline{b}}=$ $\delta_{a}^{\mu}\left(e^{-1}\right)^{a}{ }_{a}$ and we have

${ }_{a} \Omega \Gamma^{\mu}=\left[\Gamma^{\mu}-2 \delta_{a}^{\mu} \Gamma^{a}\right]{ }_{a} \Omega$.

For $\mu=a$ and $\mu=i$ we obtain

${ }_{a} \Omega \Gamma^{a}=-\Gamma_{a}^{a} \Omega, \quad{ }_{a} \Omega \Gamma^{i}=\Gamma_{a}^{i} \Omega$.

The $\Gamma$-matrices in curved space for a diagonal metric satisfy the algebra

$\left\{\Gamma^{a}, \Gamma^{b}\right\}=2\left(G^{-1}\right)^{a b}, \quad\left\{\Gamma^{a}, \Gamma^{i}\right\}=0$,

$$
\left\{\Gamma^{i}, \Gamma^{j}\right\}=2\left(G^{-1}\right)^{i j} .
$$

We should find such an ${ }_{a} \Omega$ as anticommutes with all matrices $\Gamma^{a}$ and commutes with all matrices $\Gamma^{i}$. Let us first introduce the $\Gamma^{11}$ matrix,

$\Gamma^{11}=(i)^{\frac{D(D-1)}{2}} \frac{1}{\prod_{\mu=0}^{D-1} G_{\mu \mu}} \varepsilon_{\mu_{1} \mu_{2} \cdots \mu_{D}} \Gamma^{\mu_{1}} \Gamma^{\mu_{2}} \cdots \Gamma^{\mu_{D}}$,

where the normalization constant is chosen so that $\Gamma^{11}$ satisfies the condition $\left(\Gamma^{11}\right)^{2}=1$.

Then we define an analogy of the $\Gamma^{11}$ matrix in the subspace spanned by the T-dualized directions

${ }_{a} \Gamma=(i)^{\frac{d(d-1)}{2}} \prod_{i=1}^{d} \Gamma^{a_{i}}=(i)^{\frac{d(d-1)}{2}} \Gamma^{a_{1}} \Gamma^{a_{2}} \ldots \Gamma^{a_{d}}$,

so that

$\left({ }_{a} \Gamma\right)^{2}=\prod_{i=1}^{d} G^{a_{i} a_{i}}=\frac{1}{\prod_{i=1}^{d} G_{a_{i} a_{i}}}$.

Their commutation (anticommutation) relations with one $\Gamma$ matrix depend on the number of coordinates $d$, along which we perform T-dualizations. Therefore we have

$$
{ }_{a} \Gamma \Gamma^{a}=(-1)^{d+1} \Gamma^{a}{ }_{a} \Gamma, \quad{ }_{a} \Gamma \Gamma^{i}=(-1)^{d} \Gamma^{i}{ }_{a} \Gamma,
$$

which means that the solution of Eq. (2.55) is proportional to

${ }_{a} \Omega \sim{ }_{a} \Gamma\left(\Gamma^{11}\right)^{d}$.

Taking into account (2.49), ${ }_{a} \Omega^{2}=1$, we obtain

${ }_{a} \Omega=\sqrt{\prod_{i=1}^{d} G_{a_{i} a_{i}}}{ }_{a} \Gamma\left(i \Gamma^{11}\right)^{d}$.

This is a general solution. Note that for $a_{1} \cap a_{2}=0$ we have $a_{1} \Omega a_{2} \Omega=(-1)^{d_{1} d_{2}} a \Omega$, where $a=a_{1} \bigcup a_{2}$.

When the number of coordinates along which we perform T-duality is even $(d=2 k)$, we have ${ }_{a} \Omega=$ $(-1)^{\frac{d}{2}} \sqrt{\prod_{i=1}^{d} G_{a_{i} a_{i}}} a_{a} \Gamma$. As a consequence of the relation $\Gamma^{11}{ }_{a} \Omega=(-1)^{d}{ }_{a} \Omega \Gamma^{11}$ we can conclude that in that case bar spinors preserve chirality. When the number of coordinates along which we perform T-duality is odd $(d=2 k+1)$, we have $a=(-1)^{\frac{d-1}{2}} \sqrt{\prod_{i=1}^{d} G_{a_{i} a_{i}}} i_{a} \Gamma \Gamma^{11}$. As a consequence of the above relation such a transformation changes the chirality of the bar spinors.

In the particular case that we perform T-dualization along only one direction, $x^{a_{1}},{ }_{a} \Gamma \rightarrow \Gamma^{a_{1}}, d \rightarrow 1$ and we obtain the result, well known in the literature [1-3,46,47],

${ }_{a_{1}} \Omega=i \sqrt{G_{a_{1} a_{1}}} \Gamma^{a_{1}} \Gamma^{11}$.

This is the case of the transition between IIA and IIB theory, when T-duality changes the chirality of the bar spinors.

When we perform T-dualization along all coordinates, $d \rightarrow D=10,{ }_{a} \Gamma \rightarrow \frac{\Gamma^{11}}{\sqrt{\prod_{\mu=0}^{D-1} G_{\mu \mu}}}$ and from (2.62) we obtain

${ }^{\star} \Omega=(-1)^{\frac{D}{2}} \Gamma^{11}=-\Gamma^{11}$.

2.4 Choice of the proper fermionic coordinates and T-dual background fields

We have already learned that in order to have compatibility between supersymmetry and T-duality, we should choose the dual bar variables with a bullet in accordance with (2.48). Therefore, before we read the T-dual background fields, we will reexpress the action (2.20) in terms of the appropriate spinor coordinates (2.48) which, with the help of the relation ${ }_{a} \Omega^{2}=1$, produces

$$
\begin{aligned}
{ }_{a} S & \left(y_{a}, x^{i}, \theta^{\alpha}, \bar{\theta}^{\alpha}, \pi_{\alpha}, \bar{\pi}_{\alpha}\right) \\
= & \int_{\Sigma} \mathrm{d}^{2} \xi\left\{\frac{\kappa^{2}}{2} \partial_{+} y_{a} \hat{\theta}_{-}^{a b} \partial_{-} y_{b}+\kappa^{2} \partial_{+} y_{a} \hat{\theta}_{-}^{a b} \Pi_{+b j} \partial_{-} x^{j}\right. \\
& -\kappa^{2} \partial_{+} x^{i} \Pi_{+i a} \hat{\theta}_{-}^{a b} \partial_{-} y_{b}+\frac{1}{4 \pi} \Phi R^{(2)} \\
& +\kappa \partial_{+} x^{i}\left(\Pi_{+i j}-2 \kappa \Pi_{+i a} \hat{\theta}_{-}^{a b} \Pi_{+b j}\right) \partial_{-} x^{j} \\
& -\pi_{\alpha} \partial_{-}\left(\theta^{\alpha}+\Psi_{i}^{\alpha} x^{i}-2 \Psi_{a}^{\alpha} \hat{\theta}_{-}^{a b} \Pi_{+b j} x^{j}-\Psi_{a}^{\alpha} \hat{\theta}_{-}^{a b} y_{b}\right) \\
& +\partial_{+}\left[{ }^{\bullet} \bar{\theta}^{\gamma}{ }_{a} \Omega_{\gamma}^{\alpha}+\bar{\Psi}_{i}^{\alpha} x^{i}+2 \bar{\Psi}_{a}^{\alpha} \hat{\theta}_{+}^{a b} \Pi_{-b j} x^{j}\right. \\
& \left.+\bar{\Psi}_{a}^{\alpha} \hat{\theta}_{+}^{a b} y_{b}\right]_{a} \Omega_{\alpha}{ }^{\beta} \bar{\pi}_{\beta}+2 \pi_{\alpha} \Psi_{a}^{\alpha} \hat{\theta}_{-}^{a b} \bar{\Psi}_{b a}^{\beta} \Omega_{\beta}{ }^{\gamma \bullet \bar{\pi}_{\gamma}} \\
& \left.+\frac{1}{2 \kappa} e^{\frac{\Phi}{2}} \pi_{\alpha} F^{\alpha \beta}{ }_{a} \Omega_{\beta}{ }^{\gamma} \bar{\pi}_{\gamma}\right\} .
\end{aligned}
$$

Consequently, applying the Buscher T-dualization procedure $[4,5]$ along the bosonic coordinates $x^{a}$ of the action (2.13) the T-dual action gets the form

$$
\begin{aligned}
{ }_{a} S & =\int_{\Sigma} \mathrm{d}^{2} \xi\left[\kappa \partial_{+}\left({ }_{a} X\right)_{\hat{\mu} a} \Pi_{+}^{\hat{\mu} \hat{v}} \partial_{-}\left({ }_{a} X\right)_{\hat{v}}+\frac{1}{4 \pi}{ }_{a} \Phi R^{(2)}\right. \\
& -\pi_{\alpha} \partial_{-}\left[\theta^{\alpha}+{ }_{a} \Psi^{\alpha \hat{\mu}}\left({ }_{a} X\right)_{\hat{\mu}}\right]+\partial_{+}\left[{ }^{\bullet} \bar{\theta}^{\alpha}+{ }_{a} \bar{\Psi}^{\alpha \hat{\mu}}\left({ }_{a} X\right)_{\hat{\mu}}\right]^{\bullet} \bar{\pi}_{\alpha} \\
& \left.+\frac{1}{2 \kappa} e^{\frac{a \Phi}{2}} \pi_{\alpha} a F^{\alpha \beta} \bullet_{\bar{\pi}_{\beta}}\right],
\end{aligned}
$$


where $\left({ }_{a} X\right) \hat{\mu}=\left(y_{a}, x^{i}\right),{ }_{a} \Psi^{\alpha \hat{\mu}}=\left({ }_{a} \Psi^{\alpha a},{ }_{a} \Psi_{i}^{\alpha}\right)$ and ${ }_{a} \bar{\Psi}^{\alpha \hat{\mu}}=\left({ }_{a} \bar{\Psi}^{\alpha a},{ }_{a} \bar{\Psi}_{i}^{\alpha}\right)$.

Now, we are ready to read the T-dual background fields

${ }_{a} \Pi_{ \pm}^{a b}=\frac{\kappa}{2} \hat{\theta}_{\mp}^{a b}$,

${ }_{a} \Pi_{ \pm i}{ }^{a}=-\kappa \Pi_{ \pm i b} \hat{\theta}_{\mp}^{b a}, \quad{ }_{a}\left(\Pi_{ \pm}\right)^{a}{ }_{i}=\kappa \hat{\theta}_{\mp}^{a b} \Pi_{ \pm b i}$,

${ }_{a} \Pi_{ \pm i j}=\Pi_{ \pm i j}-2 \kappa \Pi_{ \pm i a} \hat{\theta}_{\mp}^{a b} \Pi_{ \pm b j}$,

${ }_{a} \Psi^{\alpha a}=\kappa \hat{\theta}_{+}^{a b} \Psi_{b}^{\alpha}, \quad{ }_{a} \bar{\Psi}^{\alpha a}=\kappa_{a} \Omega^{\alpha}{ }_{\beta} \hat{\theta}_{-}^{a b} \bar{\Psi}_{b}^{\beta}$,

${ }_{a} \Psi_{i}^{\alpha}=\Psi_{i}^{\alpha}-2 \kappa \Pi_{-i b} \hat{\theta}_{+}^{b a} \Psi_{a}^{\alpha}$,

${ }_{a} \bar{\Psi}_{i}^{\alpha}={ }_{a} \Omega^{\alpha}{ }_{\beta}\left(\bar{\Psi}_{i}^{\beta}-2 \kappa \Pi_{+i b} \hat{\theta}_{-}^{b a} \bar{\Psi}^{\beta}\right)$,

$e^{\frac{a \Phi}{2}}{ }_{a} F^{\alpha \beta}=\left(e^{\frac{\Phi}{2}} F^{\alpha \gamma}+4 \kappa \Psi_{a}^{\alpha} \hat{\theta}_{-}^{a b} \bar{\Psi}_{b}^{\gamma}\right){ }_{a} \Omega_{\gamma}{ }^{\beta}$

when ${ }_{a} \Omega$ is defined in (2.62).

The dilaton transformation in the term $\Phi R^{(2)}$ originates from quantum theory and will be discussed in Sect. 2.6.

\subsection{Supersymmetry transformations of T-dual gravitinos}

Note that in the expressions for the T-dual fields ${ }_{a} \bar{\Psi}^{\alpha a},{ }_{a} \bar{\Psi}_{i}^{\alpha}$ and ${ }_{a} F^{\alpha \beta}$ the matrix ${ }_{a} \Omega$ appears as a consequence of the T-dualization procedure and adoption of the bullet spinor coordinates. In Refs. [46,47] it appears as a consequence of the compatibility between supersymmetry and T-duality.

A supersymmetry transformation of the gravitino is expressed in terms of covariant derivatives,

$\delta_{\varepsilon} \Psi_{\mu}^{\alpha}=D_{\mu} \varepsilon^{\alpha}+\cdots, \quad \delta_{\bar{\varepsilon}} \bar{\Psi}_{\mu}^{\alpha}=D_{\mu} \bar{\varepsilon}^{\alpha}+\cdots$,

with the same covariant derivative on both left and right spinors,

$D_{\mu}=\partial_{\mu}+\omega_{\mu}$.

In the T-dual theory, as a consequence of the two kinds of spin connections, there are two kinds of covariant derivatives,

${ }_{a} D^{\hat{\mu}}=\partial^{\hat{\mu}}+{ }_{a} \omega^{\hat{\mu}}, \quad{ }_{a} \bar{D}^{\hat{\mu}}=\partial^{\hat{\mu}}+{ }_{a} \bar{\omega}^{\hat{\mu}}$,

such that

${ }_{a} \delta_{\varepsilon}{ }_{a} \Psi^{\alpha \hat{\mu}}={ }_{a} D^{\hat{\mu}} \varepsilon^{\alpha}, \quad{ }_{a} \bar{\delta}_{\bar{\varepsilon} a} \bar{\Psi}^{\alpha \hat{\mu}}={ }_{a} \bar{D}^{\hat{\mu}} \bar{\varepsilon}^{\alpha}$.

Let us show that improvement with ${ }_{a} \Omega$ in the transformation of the bar gravitinos just turns ${ }_{a} \bar{D}^{\hat{\mu}}$ to ${ }_{a} D^{\hat{\mu}}$. In fact, from

${ }_{a} \bar{\delta}_{\bar{\varepsilon} a} \bar{\Psi}^{\alpha \hat{\mu}}={ }_{a} \Omega^{\alpha}{ }_{\beta}\left(\partial^{\hat{\mu}} \bar{\varepsilon}^{\beta}+{ }_{a} \bar{\omega}^{\hat{\mu} \beta}{ }_{\gamma} \bar{\varepsilon}^{\gamma}\right)$,

with the help of (2.43), for constant ${ }_{a} \Omega$, we have

$$
\begin{aligned}
& { }_{a} \bar{\delta}_{\bar{\varepsilon}}{ }_{a} \bar{\Psi}^{\alpha \hat{\mu}}=\partial^{\hat{\mu}}\left({ }_{a} \Omega^{\alpha}{ }_{\beta} \bar{\varepsilon}^{\beta}\right)+{ }_{a} \omega^{\hat{\mu} \alpha}{ }_{\beta}{ }_{a} \Omega^{\beta}{ }_{\gamma} \bar{\varepsilon}^{\gamma} \\
& ={ }_{a} D^{\hat{\mu}}\left({ }_{a} \Omega^{\alpha}{ }_{\beta} \bar{\varepsilon}^{\beta}\right)={ }_{a} \delta_{a} \Omega \bar{\varepsilon}{ }_{a} \bar{\Psi}^{\alpha \hat{\mu}} \text {. }
\end{aligned}
$$

Therefore, it is clear that in order to preserve the same spin connection for the two chiralities we should additionally change the bar supersymmetry parameter

$\bullet \bar{\varepsilon}^{\alpha} \equiv\left({ }_{a} \Omega\right)^{\alpha}{ }_{\beta}{ }_{a} \bar{\varepsilon}^{\beta}$.

\subsection{Transformation of pure spinors}

In this subsection we will find transformation laws for pure spinors, $\lambda^{\alpha}$ and $\bar{\lambda}^{\alpha}$, which are the main ingredient of the pure spinor formalism.

It is well known that pure spinors satisfy the so-called pure spinor constraints,

$\lambda^{\alpha}\left(\Gamma^{\mu}\right)_{\alpha \beta} \lambda^{\beta}=0, \quad \bar{\lambda}^{\alpha}\left(\Gamma^{\mu}\right)_{\alpha \beta} \bar{\lambda}^{\beta}=0$.

After T-dualization they turn into

${ }_{a} \lambda^{\alpha}\left({ }_{a} \Gamma_{\hat{\mu}}\right)_{\alpha \beta a} \lambda^{\beta}=0, \quad{ }_{a} \bar{\lambda}^{\alpha}\left({ }_{a} \bar{\Gamma}_{\hat{\mu}}\right)_{\alpha \beta}{ }_{a} \bar{\lambda}^{\beta}=0$.

The relation between matrices ${ }_{a} \Gamma_{\hat{\mu}}$ and ${ }_{a} \bar{\Gamma}_{\hat{\mu}}$ is given in (2.36). In order to have the two cases expressed with the same gamma matrices, as before, we preserve the expression for the unbar variables,

${ }_{a} \lambda^{\alpha}=\lambda^{\alpha}$

and change bar variables

$\cdot \bar{\lambda}^{\alpha}={ }_{a} \Omega^{\alpha}{ }_{\beta}{ }_{a} \bar{\lambda}^{\beta}$.

The variables $w_{\alpha}$ and $\bar{w}_{\alpha}$ are canonically conjugated momenta to the pure spinors $\lambda^{\alpha}$ and $\bar{\lambda}^{\alpha}$, respectively. The transformation laws for pure spinor momenta can be found from the expressions for $N_{+}^{\mu \nu}$ and $\bar{N}_{-}^{\mu \nu}$ (2.5) which would appear in the action if we would take higher power terms in $\theta^{\alpha}$ and $\bar{\theta}^{\alpha}$. After T-dualization these expressions become

${ }_{a} N_{+\hat{\mu} \hat{\nu}}=\frac{1}{2}{ }_{a} w_{\alpha}\left({ }_{a} \Gamma_{[\hat{\mu} \hat{\nu}]}\right)^{\alpha}{ }_{\beta}{ }_{a} \lambda^{\beta}$,

${ }_{a} \bar{N}_{-\hat{\mu} \hat{\nu}}=\frac{1}{2}{ }_{a} \bar{w}_{\alpha}\left({ }_{a} \bar{\Gamma}_{[\hat{\mu} \hat{\nu}]}\right)^{\alpha}{ }_{\beta} a_{a} \bar{\lambda}^{\beta}$.

Using Eq. (2.36) and the definition of $\Gamma^{[\mu \nu]}$ (2.16) we see that the relation between ${ }_{a} \Gamma_{[\hat{\mu} \hat{\nu}]}$ and ${ }_{a} \bar{\Gamma}_{[\hat{\mu} \hat{\nu}]}$ is the same as between the gamma matrices (2.36). As in the previous case, in order to have unique set of gamma matrices, we do not change the unbar variables,

${ }_{a} w_{\alpha}=w_{\alpha}$,

while we choose bar variables in the form

$\bullet \bar{w}_{\alpha}={ }_{a} \Omega_{\alpha}{ }^{\beta}{ }_{a} \bar{w}_{\beta}$.

Let us note that free field actions $S_{\lambda}$ and $S_{\bar{\lambda}}$ are invariant under T-dualization because ${ }_{a} \Omega^{2}=1$. 
2.7 T-dual transformation of antisymmetric fields: from IIB to IIA theory

To find the T-dual transformation laws for antisymmetric fields we will start with Eq. (2.72). First, as explained in Refs. $[4,5,60]$ the quantization procedure produces the wellknown shift in the dilaton transformation

${ }_{a} \Phi=\Phi-\ln \operatorname{det}\left(2 \Pi_{+a b}\right)=\Phi-\ln \sqrt{\frac{\operatorname{det} G_{a b}}{\operatorname{det}{ }_{a} G^{a b}}}$.

Together with (2.72) it gives a relation between the initial and T-dual background fields,

${ }_{a} F^{\alpha \beta}=\sqrt[4]{\frac{\operatorname{det} G_{a b}}{\operatorname{det}_{a} G^{a b}}}\left(F^{\alpha \gamma}+4 e^{-\frac{\Phi}{2}} \kappa \Psi_{a}^{\alpha} \hat{\theta}_{-}^{a b} \bar{\Psi}_{b}^{\gamma}\right)_{a} \Omega_{\gamma}{ }^{\beta}$.

For $B_{\mu \nu}=0$ we have ${ }_{a} G^{a b}=\left(G_{E}^{-1}\right)^{a b}=\left(G^{-1}\right)^{a b}$, and consequently $\sqrt[4]{\frac{\operatorname{det} G_{a b}}{\operatorname{det} G^{a b}}}=\sqrt[4]{\left(\operatorname{det} G_{a b}\right)^{2}}=\sqrt{\left|\operatorname{det} G_{a b}\right|}$. It is important to stress that unlike in Eq. (2.62) for ${ }_{a} \Omega$ here we have the absolute value under the square root. For a diagonal metric $G_{\mu \nu}$ we have $\operatorname{det} G_{a b}=\prod_{i=1}^{d} G_{a_{i} a_{i}}$ and taking into account Eq. (2.62) we find

$$
\begin{aligned}
{ }_{a} F^{\alpha \beta}= & i^{d} \sqrt{\operatorname{sign}\left(\prod_{i=1}^{d} G_{a_{i} a_{i}}\right)} \prod_{i=1}^{d} G_{a_{i} a_{i}} \\
& \times\left(F^{\alpha \gamma}+4 e^{-\frac{\Phi}{2}} \kappa \Psi_{a}^{\alpha} \hat{\theta}_{-}^{a b} \bar{\Psi}_{b}^{\gamma}\right)\left({ }_{a} \Gamma \Gamma_{11}^{d}\right)_{\gamma}{ }^{\beta} .
\end{aligned}
$$

Note that we are going to T-dualize all $D$-directions. Then it is necessary to perform T-dualization along the time-like direction. Here the above square root has important consequences. For our signature $(+,-,-, \ldots,-)$, the square of the field strength $\left({ }_{a} F^{\alpha \beta}\right)^{2}$ and, consequently, the square of all antisymmetric fields will change the sign when we perform T-dualization along the time-like direction. This is just what we need to obtain type $I I^{\star}$ theories in accordance with Ref. [37].

In a simple case when gravitino fields and Kalb-Ramond field are zero and metric is diagonal we will express the transition from type IIB to type IIA theory. Taking $d=1$ we have

${ }_{a} F^{\alpha \beta}=i \sqrt{\operatorname{sign}\left(G_{a a}\right)} G_{a a} F^{\alpha \gamma}\left(\Gamma^{11} \Gamma^{a}\right) \gamma^{\beta}$.

Let us choose type IIB as a starting theory. The matrix $\Gamma^{11}$ turns $F^{(n)}$ to $F^{(10-n)}$ where

$\left.\left(\mathrm{F}^{(n)}\right)^{\alpha \beta}=\frac{1}{n !} F_{\mu_{1} \mu_{2} \cdots \mu_{n}}\left(\Gamma^{\left[\mu_{1} \mu_{2} \cdots \mu_{n}\right.}\right]\right)^{\alpha \beta}$.

As a consequence of the chirality condition $F \Gamma^{11}=-\Gamma^{11} F$ the independent tensors are $F^{(1)}, F^{(3)}$ and self-dual part of
$F^{(5)}$. So we can write

$F^{\alpha \gamma}\left(\Gamma^{11}\right)_{\gamma}^{\beta}=\left(\mathrm{F}^{(1)}+\mathrm{F}^{(3)}+\frac{1}{2} \mathrm{~F}^{(5)}\right)^{\alpha \beta}$

Similarly, in T-dual theory (here it is IIA) we have

${ }_{a} F^{\alpha \beta}=\left({ }_{a} \mathrm{~F}^{(2)}+{ }_{a} \mathrm{~F}^{(4)}\right)^{\alpha \beta}$,

where now

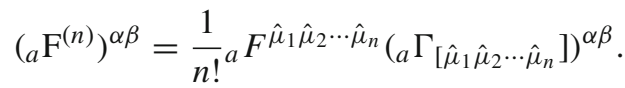

The $\Gamma$-matrices on both sides are defined in curved space. For the initial theory it is just (2.52), while for T-dual theory it is defined in the first relation in Eq. (2.35) as ${ }_{a} \Gamma_{\hat{\mu}}=$ $\left({ }_{a} e^{-1}\right)_{\hat{\mu} a} \Gamma^{\underline{a}}$. As a consequence of the first relation (2.29) between the vielbeins ${ }_{a} e^{\underline{a} \hat{\mu}}=e^{\underline{a}}{ }_{\nu}\left(Q^{T}\right)^{v \hat{\mu}}$ we can find the relation between the $\Gamma$-matrices,

${ }_{a} \Gamma_{\hat{\mu}}=\left(Q^{-1 T}\right)_{\hat{\mu} v} \Gamma^{v}$,

which produces

$\left({ }_{a} \mathrm{~F}^{(n)}\right)^{\alpha \beta}=\frac{1}{n !}\left(\frac{Q}{a} F\right)_{\mu_{1} \mu_{2} \cdots \mu_{n}}\left(\Gamma^{\left[\mu_{1} \mu_{2} \cdots \mu_{n}\right]}\right)^{\alpha \beta}$,

where

$$
\begin{aligned}
\left({ }_{a}^{Q} F\right)_{\mu_{1} \mu_{2} \cdots \mu_{n}}= & { }_{a} F^{\hat{\mu}_{1} \hat{\mu}_{2} \cdots \hat{\mu}_{n}}\left(Q^{-1 T}\right)_{\hat{\mu}_{1} \mu_{1}} \\
& \times\left(Q^{-1 T}\right)_{\hat{\mu}_{2} \mu_{2}} \cdots\left(Q^{-1 T}\right)_{\hat{\mu}_{n} \mu_{n}} .
\end{aligned}
$$

Using the standard relation between the $\Gamma$-matrices,

$\Gamma^{\left[\mu_{1} \mu_{2} \cdots \mu_{n}\right]} \Gamma^{a}=\Gamma^{\mu \mu_{1} \mu_{2} \cdots \mu_{n} a}-\frac{1}{(n-1) !} G^{a\left[\mu_{n}\right.} \Gamma^{\left.\mu_{1} \mu_{2} \cdots \mu_{n-1}\right]}$,

we obtain

$$
\begin{aligned}
F^{(n)} \Gamma^{a}= & \frac{1}{n !} F_{\mu_{1} \mu_{2} \cdots \mu_{n}} \Gamma^{\left[\mu_{1} \mu_{2} \cdots \mu_{n} a\right]} \\
& -\frac{1}{(n-1) !} F_{\mu_{1} \mu_{2} \cdots \mu_{n-1}}{ }^{a} \Gamma^{\left[\mu_{1} \mu_{2} \cdots \mu_{n-1}\right]} .
\end{aligned}
$$

Therefore, from (2.90), (2.92), (2.93), (2.96), (2.97) and (2.99) we can find a general relation connecting antisymmetric fields of Type IIA and type IIB theories,

$$
\begin{aligned}
& { }_{a} \mathrm{~F}^{\hat{\mu}_{1} \hat{\mu}_{2} \cdots \hat{\mu}_{n}}=\sqrt{\operatorname{sign} G_{a a}} G_{a a}\left(n F_{\mu_{1} \mu_{2} \cdots \mu_{n-1}} \delta^{a}{ }_{\mu_{n}}\right. \\
& \left.\quad-F_{\mu_{1} \mu_{2} \cdots \mu_{n}}{ }^{a}\right)\left(Q^{T}\right)^{\mu_{1} \hat{\mu}_{1}}\left(Q^{T}\right)^{\mu_{2} \hat{\mu}_{2}} \cdots\left(Q^{T}\right)^{\mu_{n} \hat{\mu}_{n}} .
\end{aligned}
$$

Under our assumptions we have

$\left(Q^{T}\right)^{\mu \hat{\mu}}=\left(\begin{array}{cc}-G^{a a} & 0 \\ 0 & \delta_{i}^{j}\end{array}\right)$ 
and consequently

$$
{ }_{a} F_{i j}=-i \sqrt{\operatorname{sign} G_{a a}} G_{a a} F_{i j}{ }^{a}, \quad{ }_{a} F_{i}^{a}=-2 i \sqrt{\operatorname{sign} G_{a a}} F_{i},
$$

${ }_{a} F_{i j k q}=-\frac{i}{2} \sqrt{\operatorname{sign} G_{a a}} G_{a a} F_{i j k q}{ }^{a}$,

${ }_{a} F_{i j k}{ }^{a}=-4 i \sqrt{\operatorname{sign} G_{a a}} F_{i j k}$.

For the space-like directions $G_{a a}<0$ and $i \sqrt{\operatorname{sign} G_{a a}}$ is real. For time-like direction $\sqrt{\operatorname{sign} G_{a a}} \rightarrow \sqrt{\operatorname{sign} G_{00}}=1$ and the remaining imaginary unit causes squares of the antisymmetric fields to get an additional minus sign and type II theories to swap to type $\mathrm{II}^{\star}$ ones [37].

\section{Double space formulation}

In this section we will introduce double space, doubling all bosonic coordinates $x^{\mu}$ by corresponding T-dual ones $y_{\mu}$. We will rewrite the transformation laws in double space and show that both the equations of motion and the Bianchi identities can be written by that single equation.

\subsection{T-dualization along all bosonic directions}

Applying the Buscher T-dualization procedure [4,5] along all bosonic coordinates of the action (2.13) the T-dual action has been obtained in Ref. [9]. This is a particular case of our relations (2.67)-(2.72) where the T-dual background fields are of the form

$$
\begin{aligned}
& { }^{\star} \Pi_{ \pm}^{\mu \nu} \equiv{ }^{\star} B^{\mu \nu} \pm \frac{1}{2}{ }^{\star} G^{\mu \nu}=\frac{\kappa}{2} \Theta_{\mp}^{\mu \nu}, \\
& { }^{\star} \Psi^{\alpha \mu}=\kappa \Theta_{+}^{\mu \nu} \Psi_{v}^{\alpha}, \quad{ }^{\star} \bar{\Psi}^{\alpha \mu}=\kappa^{\star} \Omega^{\alpha}{ }_{\beta} \Theta_{-}^{\mu \nu} \bar{\Psi}_{v}^{\beta}, \\
& e^{{ }^{\star} \Phi}{ }^{\star} F^{\alpha \beta}=\left(e^{\frac{\Phi}{2}} F^{\alpha \gamma}+4 \kappa \Psi_{\mu}^{\alpha} \Theta_{-}^{\mu \nu} \bar{\Psi}_{\nu}^{\gamma}\right){ }^{\star} \Omega_{\gamma}{ }^{\beta} .
\end{aligned}
$$

Here we use the notation

$$
\begin{aligned}
G_{\mu \nu}^{E} & =G_{\mu \nu}-4\left(B G^{-1} B\right)_{\mu \nu}, \Theta^{\mu \nu}=-\frac{2}{\kappa}\left(G_{E}^{-1} B G^{-1}\right)^{\mu \nu}, \\
{ }^{\star} \Omega & =-\Gamma^{11},
\end{aligned}
$$

and

$\Theta_{ \pm}^{\mu \nu}=-\frac{2}{\kappa}\left(G_{E}^{-1} \Pi_{ \pm} G^{-1}\right)^{\mu \nu}=\Theta^{\mu \nu} \mp \frac{1}{\kappa}\left(G_{E}^{-1}\right)^{\mu \nu}$,

so that

$\left(\Pi_{ \pm} \Theta_{\mp}\right)_{\mu}{ }^{v}=\frac{1}{2 \kappa} \delta_{\mu}{ }^{v}$.

From (3.1) and (3.5) it follows that

${ }^{\star} G^{\mu \nu}=\left(G_{E}^{-1}\right)^{\mu \nu}, \quad{ }^{\star} B^{\mu \nu}=\frac{\kappa}{2} \Theta^{\mu \nu}$.

In this case the transformation laws (2.21) and (2.22) (the relations between the initial $x^{\mu}$ and T-dual coordinates $y_{\mu}$ ) get the form

$$
\begin{gathered}
\partial_{ \pm} x^{\mu} \cong-\kappa \Theta_{ \pm}^{\mu \nu} \partial_{ \pm} y_{\nu}+\kappa \Theta_{ \pm}^{\mu \nu} J_{ \pm \nu} \\
\partial_{ \pm} y_{\mu} \cong-2 \Pi_{\mp \mu \nu} \partial_{ \pm} x^{\nu}+J_{ \pm \mu}
\end{gathered}
$$

\subsection{Transformation laws in double space}

Rewriting Eq. (3.8) in the form where terms multiplied by $\varepsilon_{ \pm}{ }^{ \pm}= \pm 1$ are on the left-hand side of the equation, we obtain

$$
\begin{aligned}
& \pm \partial_{ \pm} y_{\mu} \cong G_{E \mu \nu} \partial_{ \pm} x^{\nu}-2\left(B G^{-1}\right)_{\mu}{ }^{\nu} \partial_{ \pm} y_{v} \\
& \quad+2\left(\Pi_{ \pm} G^{-1}\right)_{\mu}{ }^{\nu} J_{ \pm v}, \\
& \pm \partial_{ \pm} x^{\mu} \cong\left(G^{-1}\right)^{\mu \nu} \partial_{ \pm} y_{v} \\
& \quad+2\left(G^{-1} B\right)^{\mu}{ }_{\nu} \partial_{ \pm} x^{\nu}-\left(G^{-1}\right)^{\mu \nu} J_{ \pm v} .
\end{aligned}
$$

Let us introduce double space coordinates

$Z^{M}=\left(\begin{array}{c}x^{\mu} \\ y_{\mu}\end{array}\right)$

which contain all initial and T-dual coordinates. In terms of double coordinates Eqs. (3.9) and (3.10) are replaced by one equation:

$\partial_{ \pm} Z^{M} \cong \pm \Omega^{M N}\left(\mathcal{H}_{N P} \partial_{ \pm} Z^{P}+J_{ \pm N}\right)$,

where the matrix $\mathcal{H}_{M N}$ is known in the literature as the generalized metric and has the form

$\mathcal{H}_{M N}=\left(\begin{array}{cc}G_{\mu \nu}^{E} & -2 B_{\mu \rho}\left(G^{-1}\right)^{\rho \nu} \\ 2\left(G^{-1}\right)^{\mu \rho} B_{\rho \nu} & \left(G^{-1}\right)^{\mu \nu}\end{array}\right)$.

The double current $J_{ \pm M}$ is defined as

$J_{ \pm M}=\left(\begin{array}{c}2\left(\Pi_{ \pm} G^{-1}\right)_{\mu}^{\nu} J_{ \pm v} \\ -\left(G^{-1}\right)^{\mu \nu} J_{ \pm v}\end{array}\right)$

and

$\Omega^{M N}=\left(\begin{array}{cc}0 & 1_{D} \\ 1_{D} & 0\end{array}\right)$

is a constant symmetric matrix. Here $1_{D}$ denotes the identity operator in $D$ dimensions. Let us stress that the matrix ${ }_{a} \Omega$ and $\Omega^{M N}$ are different quantities.

By straightforward calculation we can prove the relations

$\mathcal{H}^{T} \Omega \mathcal{H}=\Omega, \quad \Omega^{2}=1, \quad \operatorname{det} \mathcal{H}_{M N}=1$,

which means that $\mathcal{H} \in S O(D, D)$. In calculation of determinant we use the rule for block matrices

$\operatorname{det}\left(\begin{array}{ll}A & B \\ C & D\end{array}\right)=\operatorname{det} D \operatorname{det}\left(A-B D^{-1} C\right)$.

In double field theory $\Omega^{M N}$ is the $S O(D, D)$ invariant metric and denoted by $\eta^{M N}$. 
3.3 Equations of motion and double space action

It is well known that the equations of motion of the initial theory are the Bianchi identities in T-dual picture and vice versa $[12,19,22,60]$. As a consequence of the identity

$\partial_{+} \partial_{-} Z^{M}-\partial_{-} \partial_{+} Z^{M}=0$,

known as the Bianchi identity, and Eq. (3.12), we obtain the consistency condition

$\partial_{+}\left[\mathcal{H}_{M N} \partial_{-} Z^{N}+J_{-M}\right]+\partial_{-}\left[\mathcal{H}_{M N} \partial_{+} Z^{N}+J_{+M}\right]=0$.

In components it takes the form

$$
\begin{aligned}
& \partial_{+} \partial_{-} x^{\mu}=-\frac{1}{\kappa}\left(G^{-1}\right)^{\mu \nu}\left(\bar{\Psi}_{\nu}^{\alpha} \partial_{+} \bar{\pi}_{\alpha}+\Psi_{\mu}^{\alpha} \partial_{-} \pi_{\alpha}\right), \\
& \partial_{+} \partial_{-} y_{\nu}=-\frac{1}{\kappa} G_{\mu \nu}^{E}\left({ }^{\star} \bar{\Psi}^{\alpha \mu} \partial_{+} \bar{\pi}_{\alpha}+{ }^{\star} \Psi^{\alpha \mu} \partial_{-} \pi_{\alpha}\right) .
\end{aligned}
$$

These equations are equations of motion of the initial and T-dual theory. Double space formalism enables us to write both equations of motion and Bianchi identities by the single relation (3.12).

Equation (3.19) is the equation of motion of the following action:

$$
\begin{aligned}
S= & \frac{\kappa}{4} \int \mathrm{d}^{2} \xi\left[\partial_{+} Z^{M} \mathcal{H}_{M N} \partial_{-} Z^{N}+\partial_{+} Z^{M} J_{-M}\right. \\
& \left.+J_{+M} \partial_{-} Z^{M}+L\left(\pi_{\alpha}, \bar{\pi}_{\alpha}\right)\right],
\end{aligned}
$$

where $L\left(\pi_{\alpha}, \bar{\pi}_{\alpha}\right)$ is an arbitrary functional of the fermionic momenta.

\section{T-dualization of type II superstring theory as a permutation of coordinates in double space}

In this section we will derive the transformations of the generalized metric and current, which are a consequence of the permutation of some subset of the bosonic coordinates with the corresponding T-dual ones. First we will present the method in the case of the complete T-dualization (along all bosonic coordinates) and find the expressions for T-dual background fields. Then we will apply the results to the case of partial T-dualization.

\subsection{The case of complete T-dualization}

In order to exchange all initial and T-dual coordinates let us introduce the permutation matrix

$\mathcal{T}^{M}{ }_{N}=\left(\begin{array}{cc}0 & 1_{D} \\ 1_{D} & 0\end{array}\right)$, so that the double T-dual coordinate ${ }^{\star} Z^{M}$ is obtained:

${ }^{\star} Z^{M}=\mathcal{T}^{M}{ }_{N} Z^{N}=\left(\begin{array}{c}y_{\mu} \\ x^{\mu}\end{array}\right)$.

We require that the T-dual transformation law for the double T-dual coordinate ${ }^{\star} Z^{M}$ has the same form as for the initial coordinate $Z^{M}$ (3.12)

$\partial_{ \pm}{ }^{\star} Z^{M} \cong \pm \Omega^{M N}\left({ }^{\star} \mathcal{H}_{N P} \partial_{ \pm}{ }^{\star} Z^{P}+{ }^{\star} J_{ \pm N}\right)$.

Then the T-dual generalized metric ${ }^{\star} \mathcal{H}_{M N}$ and T-dual current ${ }^{\star} J_{ \pm M}$ are

${ }^{\star} \mathcal{H}_{M N}=\mathcal{T}_{M}{ }^{K} \mathcal{H}_{K L} \mathcal{T}^{L}{ }_{N}, \quad{ }^{\star} J_{ \pm M}=\mathcal{T}_{M}{ }^{N} J_{ \pm N}$.

Permutation of the coordinates (4.2) together with transformations of the background fields (4.4) represents the symmetry transformations of the action (3.21).

Using the corresponding expressions for $\mathcal{T}^{M}{ }_{N}, \mathcal{H}_{M N}$ and $J_{ \pm M}$, we obtain from the generalized metric transformation

${ }^{\star} G^{\mu \nu}=\left(G_{E}^{-1}\right)^{\mu \nu}, \quad{ }^{\star} B^{\mu \nu}=\frac{\kappa}{2} \Theta^{\mu \nu}$.

Taking into account that as a consequence of (2.48) the bar dual variable is ${ }^{\bullet \star} \bar{\pi}_{\alpha}=\left({ }^{\star} \Omega^{T}\right)_{\alpha}{ }^{\beta} \bar{\pi}_{\beta}$, from the current transformations we have

${ }^{\star} \Psi^{\alpha \mu}=\kappa \Theta_{+}^{\mu \nu} \Psi_{v}^{\alpha}, \quad{ }^{\star} \bar{\Psi}^{\alpha \mu}=\kappa{ }^{\star} \Omega^{\alpha}{ }_{\beta} \Theta_{-}^{\mu \nu} \bar{\Psi}_{v}^{\beta}$,

where ${ }^{\star} \Omega=-\Gamma^{11}$.

Consequently, using double space we can easily reproduce the results of T-dualization, Eqs. (3.7) and (3.2). The problem with T-dualization of the R-R field strength $F^{\alpha \beta}$ will be discussed in Sect. 5.3.

\subsection{The case of partial T-dualization}

Applying the procedure presented in the previous subsection to the arbitrary subset of bosonic coordinates we will, in fact, describe all possible bosonic T-dualizations. Let us split the coordinate index $\mu$ into $a$ and $i(a=0, \ldots, d-1$, $i=d, \ldots, D-1)$ and denote T-dualization along direction $x^{a}$ and $y_{a}$ by

$$
\begin{aligned}
& \mathcal{T}^{a}=T^{a} \circ T_{a}, \quad T^{a} \equiv T^{0} \circ T^{1} \circ \cdots \circ T^{d-1}, \\
& T_{a} \equiv T_{0} \circ T_{1} \circ \cdots \circ T_{d-1},
\end{aligned}
$$

where $\circ$ marks the operation of composition of T-dualizations. Permutation of the initial coordinates $x^{a}$ with its T-dual $y_{a}$ is realized by multiplying the double space coordinate by the constant symmetric matrix $\left(\mathcal{T}^{a}\right)^{M}{ }_{N}$,

${ }_{a} Z^{M} \equiv\left(\begin{array}{c}y_{a} \\ x^{i} \\ x^{a} \\ y_{i}\end{array}\right)=\left(\mathcal{T}^{a}\right)^{M}{ }_{N} Z^{N} \equiv\left(\begin{array}{cccc}0 & 0 & 1_{a} & 0 \\ 0 & 1_{i} & 0 & 0 \\ 1_{a} & 0 & 0 & 0 \\ 0 & 0 & 0 & 1_{i}\end{array}\right)\left(\begin{array}{c}x^{a} \\ x^{i} \\ y_{a} \\ y_{i}\end{array}\right)$, 
where $1_{a}$ and $1_{i}$ are identity operators in the subspaces spanned by $x^{a}$ and $x^{i}$, respectively. It is easily to check the following relations:

$$
\left(\mathcal{T}^{a} \mathcal{T}^{a}\right)^{M}{ }_{N}=\delta^{M}{ }_{N}, \quad\left(\mathcal{T}^{a} \Omega \mathcal{T}^{a}\right)^{M}{ }_{N}=\Omega^{M}{ }_{N} .
$$

The first relation means that after two T-dualizations we get the initial theory, while the second relation means that $\mathcal{T}^{a} \in$ $O(D, D)$.

Let us apply the same approach as in the case of the full Tdualization presented in the previous subsection. We require that the double T-dual coordinate ${ }_{a} Z^{M}$ satisfy the T-duality transformations of the form like the initial one $Z^{M}$ (3.12),

$\partial_{ \pm a} Z^{M} \cong \pm \Omega^{M N}\left({ }_{a} \mathcal{H}_{N K} \partial_{ \pm{ }_{a}} Z^{K}+{ }_{a} J_{ \pm N}\right)$.

Consequently, we find the T-dual generalized metric

${ }_{a} \mathcal{H}_{M N}=\left(\mathcal{T}^{a}\right)_{M}{ }^{K} \mathcal{H}_{K L}\left(\mathcal{T}^{a}\right)^{L}{ }_{N}$,

and the T-dual current

${ }_{a} J_{ \pm M}=\left(\mathcal{T}^{a}\right)_{M}{ }^{N} J_{ \pm N}$

Note that Eqs. (4.8), (4.11) and (4.12) are symmetry transformations of the action (3.21). The left subscript $a$ means dualization along the $x^{a}$ directions.

\section{T-dual background fields}

In this section we will show that permutation of some bosonic coordinates leads to the same T-dual background fields as standard Buscher procedure [9]. The transformation of the generalized metric (4.11) produces expressions for NS-NS T-dual background fields $\left(G_{\mu \nu}\right.$ and $\left.B_{\mu \nu}\right)$. They are the same as in bosonic string case obtained in Ref. [35]. Therefore, we will just shortly repeat these results. From the transformation of the current $J_{ \pm M}$ (4.12) we will find T-dual background fields of the NS-R sector $\left(\Psi_{\mu}^{\alpha}\right.$ and $\left.\bar{\Psi}_{\mu}^{\alpha}\right)$. Because R-R field strength $F^{\alpha \beta}$ does not appear in T-dual transformations, we will find its T-dual under some assumptions.

\subsection{T-dual NS-NS background fields $G_{\mu \nu}, B_{\mu \nu}$}

Requiring that the T-dual generalized metric ${ }_{a} \mathcal{H}_{M N}$ has the same form as the initial one $\mathcal{H}_{M N}$ (3.13) but in terms of the T-dual fields

${ }_{a} \mathcal{H}_{M N}=\left(\begin{array}{cc}{ }_{a} G_{E}^{\mu \nu} & -2\left({ }_{a} B{ }_{a} G^{-1}\right)^{\mu}{ }_{\nu} \\ 2\left({ }_{a} G^{-1}{ }_{a} B\right)_{\mu}{ }^{\nu} & \left({ }_{a} G^{-1}\right)_{\mu \nu}\end{array}\right)$,

and using Eq. (4.11), one finds expressions for the NS-NS T-dual background fields ${ }_{a} \Pi_{ \pm}^{\mu v}$ in terms of the initial ones, where $\gamma$ and $\bar{\gamma}$ are defined in (A.4), $g$ and $\tilde{g}$ in (A.5), while $\beta_{1}, \tilde{\beta}$ and $\bar{\beta}$ are defined in (A.7). The quantities $A$ and $D$ are given in (A.11) and (A.13), respectively. In more compact form we have

${ }_{a} \Pi_{ \pm}^{\mu v}=\left(\begin{array}{cc}\frac{\kappa}{2} \hat{\theta}_{\mp}^{a b} & \kappa \hat{\theta}_{\mp}^{a b} \Pi_{ \pm b i} \\ -\kappa \Pi_{ \pm i b} \hat{\theta}_{\mp}^{b a} & \Pi_{ \pm i j}-2 \kappa \Pi_{ \pm i a} \hat{\theta}_{\mp}^{a b} \Pi_{ \pm b j}\end{array}\right)$

where $\hat{\theta}_{ \pm}^{a b}$ has been defined in (A.9). Details regarding the derivation of the Eqs. (5.2) and (5.3) are given in Ref. [35]. Reading the block components we obtained the NS-NS Tdual background fields in the flat background after dualization along the directions $x^{a},(a=0,1, \ldots, d-1)$

${ }_{a} \Pi_{ \pm}^{a b}=\frac{\kappa}{2} \hat{\theta}_{\mp}^{a b}, \quad{ }_{a} \Pi_{ \pm i}^{a}=\kappa \hat{\theta}_{\mp}^{a b} \Pi_{ \pm b i}$,

${ }_{a} \Pi_{ \pm i}{ }^{a}=-\kappa \Pi_{ \pm i b} \hat{\theta}_{\mp}^{b a}$,

${ }_{a} \Pi_{ \pm i j}=\Pi_{ \pm i j}-2 \kappa \Pi_{ \pm i a} \hat{\theta}_{\mp}^{a b} \Pi_{ \pm b j}$.

These are just the Eqs. (2.67)-(2.69). The symmetric and antisymmetric parts of these expressions are the T-dual metric and T-dual Kalb-Ramond field, which are in full agreement with the Refs. [9,20].

\subsection{T-dual NS-R background fields $\Psi_{\mu}^{\alpha}, \bar{\Psi}_{\mu}^{\alpha}$}

Let us find the form of T-dual NS-R background fields, ${ }_{a} \Psi^{\alpha a}$, ${ }_{a} \Psi_{i}^{\alpha},{ }_{a} \bar{\Psi}^{\alpha a}$ and ${ }_{a} \bar{\Psi}_{i}^{\alpha}$. The T-dual current ${ }_{a} J_{ \pm M}$ (4.12) should have the same form as the initial one, Eq. (3.14), but in terms of the T-dual background fields

$$
\begin{aligned}
& \left(\begin{array}{c}
2\left({ }_{a} \Pi_{ \pm}{ }_{a} G^{-1}\right)^{a}{ }_{b}\left({ }_{a} J\right)_{ \pm}^{b}+2\left({ }_{a} \Pi_{ \pm}{ }_{a} G^{-1}\right)^{a i}\left({ }_{a} J\right)_{ \pm i} \\
2\left({ }_{a} \Pi_{ \pm}{ }_{a} G^{-1}\right)_{i a}\left({ }_{a} J\right)_{ \pm}^{a}+2\left({ }_{a} \Pi_{ \pm}{ }_{a} G^{-1}\right)_{i}{ }^{j}\left({ }_{a} J\right)_{ \pm j} \\
-\left({ }_{a} G^{-1}\right)_{a b}\left({ }_{a} J\right)_{ \pm}^{b}-\left({ }_{a} G^{-1}\right)_{a}{ }^{i}\left({ }_{a} J\right)_{ \pm i} \\
-\left({ }_{a} G^{-1}\right)^{i}{ }_{a}\left({ }_{a} J\right)_{ \pm}^{a}-\left({ }_{a} G^{-1}\right)^{i j} \quad\left({ }_{a} J\right)_{ \pm j}
\end{array}\right) \\
& =\left(\begin{array}{c}
-\left(G^{-1}\right)^{a \mu} J_{ \pm \mu} \\
2\left(\Pi_{ \pm} G^{-1}\right)_{i}{ }^{\mu} J_{ \pm \mu} \\
2\left(\Pi_{ \pm} G^{-1}\right)_{a}{ }^{\mu} J_{ \pm \mu} \\
-\left(G^{-1}\right)^{i \mu} J_{ \pm \mu}
\end{array}\right) .
\end{aligned}
$$

On the left-hand side of this equation we split the index $\mu$ in $a$ and $i$ components because in the T-dual picture the index $a$ has a different position, it is now up. T-dual currents are written between the brackets to make a distinction between a left subscript $a$ denoting partial T-dualization and summation indices in the subspace spanned by $x^{a}$.

We can obtain the information about T-dual NS-R background fields from the lower $D$ components of the above equation. In order to find the solution of these equations it is more practical to rewrite them using the block-wise form of matrices given in the appendix and Ref. [35],

${ }_{a} \Pi_{ \pm}^{\mu \nu}=\left(\begin{array}{cc}\tilde{g}^{-1} \beta_{1} D^{-1} \gamma-A^{-1}\left(\tilde{\beta} \mp \frac{1}{2}\right) & \frac{1}{2} A^{-1} g^{T}-2 \tilde{g}^{-1} \beta_{1} D^{-1}\left(\bar{\beta}^{T} \mp \frac{1}{2}\right) \\ \frac{1}{2} D^{-1} \gamma-2 \bar{\gamma}^{-1} \beta_{1}^{T} A^{-1}\left(\tilde{\beta} \mp \frac{1}{2}\right) & \bar{\gamma}^{-1} \beta_{1}^{T} A^{-1} g^{T}-D^{-1}\left(\bar{\beta}^{T} \mp \frac{1}{2}\right)\end{array}\right)$ 


$$
\begin{aligned}
& -\tilde{g}_{a b}\left({ }_{a} J\right)_{ \pm}^{b}+2\left(\beta_{1}\right)_{a}{ }^{i}\left({ }_{a} J\right)_{ \pm i} \\
& \quad=2\left(\tilde{\beta} \pm \frac{1}{2}\right){ }_{a}{ }^{b} J_{ \pm b}+2\left(\beta_{1}\right)_{a}{ }^{i} J_{ \pm i}, \\
& -2\left(\beta_{1}^{T}\right)_{b}{ }_{b}\left({ }_{a} J\right)_{ \pm}^{b}+\bar{\gamma}^{i j}\left({ }_{a} J\right)_{ \pm j}=\gamma^{i a} J_{ \pm a}+\bar{\gamma}^{i j} J_{ \pm j} .
\end{aligned}
$$

From Eq. (3.18) of [35]

$$
\begin{aligned}
\left({ }_{a} G^{-1}\right)_{\mu \nu} & =\left(\begin{array}{cc}
g_{a b} & -2\left(B G^{-1}\right) a^{j} \\
2\left(G^{-1} B\right)^{i}{ }_{b} & \left(G^{-1}\right)^{i j}
\end{array}\right) \\
& =\left(\begin{array}{cc}
\tilde{g} & -2 \beta_{1} \\
-2 \beta_{1}^{T} & \bar{\gamma}
\end{array}\right),
\end{aligned}
$$

(A.4) and (A.7), we find the components of ${ }_{a} G^{-1}, G^{-1}$ and $B G^{-1}$, respectively. In the first equation on right-hand side for $\left(\Pi_{ \pm} G^{-1}\right)_{a}{ }^{i}$ just stands for $\left(\beta_{1}\right)_{a}{ }^{i}$ because $\delta_{a}{ }^{i}=0$.

The difference

$$
\left({ }_{a} J\right)_{ \pm i}-J_{ \pm i}=\left(\bar{\gamma}^{-1}\right)_{i j}\left[\gamma^{j a} J_{ \pm a}+2\left(\beta_{1}^{T}\right)_{b}^{j}\left({ }_{a} J\right)_{ \pm}^{b}\right]
$$

obtained from the second equation, we put in the first equation, which produces

$$
\begin{gathered}
2\left[\left(\tilde{\beta} \pm \frac{1}{2}\right)-\beta_{1} \bar{\gamma}^{-1} \gamma\right]_{a}{ }^{b} J_{ \pm b} \\
=-\left(\tilde{g}-4 \beta_{1} \bar{\gamma}^{-1} \beta_{1}^{T}\right)_{a b}{ }_{a} J_{ \pm}^{b} .
\end{gathered}
$$

From the definition of the quantity $A_{a b}$ (A.11) we get

$\left({ }_{a} J\right)_{ \pm}^{b}=2\left[-A^{-1}\left(\tilde{\beta} \pm \frac{1}{2}\right)+A^{-1} \beta_{1} \bar{\gamma}^{-1} \gamma\right]^{b c} J_{ \pm c}$.

Using the expression $A_{a b}=\hat{g}_{a b}$ (proved in [35]) and Eq. (A.12), we recognize the $a b$ block component of Eq. (5.2). Therefore, with the help of (5.3) it is easy to see that

$\left({ }_{a} J\right)_{ \pm}^{b}=2{ }_{a} \Pi_{\mp}^{b c} J_{ \pm c}=\kappa \hat{\theta}_{ \pm}^{b c} J_{ \pm c}$.

Note that now the T-dual current ${ }_{a} J_{ \pm}^{\hat{\mu}}$ is of the form

${ }_{a} J_{ \pm}^{\hat{\mu}}= \pm \frac{2}{\kappa}{ }_{a} \Psi_{ \pm}^{\alpha \hat{\mu}}{ }_{a} \pi_{ \pm \alpha}$,

where

$$
\begin{aligned}
& { }_{a} \Psi_{+}^{\alpha \hat{\mu}} \equiv{ }_{a} \Psi^{\alpha \hat{\mu}}, \quad{ }_{a} \Psi_{-}^{\alpha \hat{\mu}} \equiv{ }_{a} \bar{\Psi}^{\alpha \hat{\mu}}, \quad{ }_{a} \pi_{+\alpha} \equiv \pi_{\alpha}, \\
& { }_{a} \pi_{-\alpha} \equiv \bar{\pi}_{\alpha},
\end{aligned}
$$

and as before

$J_{ \pm \mu}= \pm \frac{2}{\kappa} \Psi_{ \pm \mu}^{\alpha} \pi_{ \pm \alpha}$.

Therefore, the $a$ components of the T-dual NS-R fields are of the form

${ }_{a} \Psi^{\alpha a}=\kappa \hat{\theta}_{+}^{a b} \Psi_{b}^{\alpha}, \quad{ }_{a} \bar{\Psi}^{\alpha a}=\kappa_{a} \Omega^{\alpha}{ }_{\beta} \hat{\theta}_{-}^{a b} \bar{\Psi}_{b}^{\beta}$.
Substituting (5.11) into (5.9) we obtain

$$
\begin{aligned}
\left({ }_{a} J\right)_{ \pm i}-J_{ \pm i}= & \left(\bar{\gamma}^{-1}+4 \bar{\gamma}^{-1} \beta_{1}^{T} A^{-1} \beta_{1} \bar{\gamma}^{-1}\right)_{i j} \gamma^{j b} J_{ \pm b} \\
& -4\left[\bar{\gamma}^{-1} \beta_{1}^{T} A^{-1}\left(\tilde{\beta} \pm \frac{1}{2}\right)\right]_{i}{ }^{a} J_{ \pm a} .
\end{aligned}
$$

With the help of (A.13) Eq. (5.17) transforms into

$$
\begin{aligned}
& \left({ }_{a} J\right)_{ \pm i}-J_{ \pm i} \\
& \quad=2\left[\frac{1}{2} D^{-1} \gamma-2 \bar{\gamma}^{-1} \beta_{1}^{T} A^{-1}\left(\tilde{\beta} \pm \frac{1}{2}\right)\right]_{i}{ }^{a} J_{ \pm a} .
\end{aligned}
$$

From the ${ }_{i}^{a}$ component of (5.2) and (5.3) we finally have

$\left({ }_{a} J\right)_{ \pm i}=J_{ \pm i}-2 \kappa \Pi_{\mp i b} \hat{\theta}_{ \pm}^{b a} J_{ \pm a}$.

As in the previous case, using the expressions for the currents (5.13) and (5.15), the final form of the T-dual fields is

$$
\begin{aligned}
& { }_{a} \Psi_{i}^{\alpha}=\Psi_{i}^{\alpha}-2 \kappa \Pi_{-i b} \hat{\theta}_{+}^{b a} \Psi_{a}^{\alpha}, \\
& \quad{ }_{a} \bar{\Psi}_{i}^{\alpha}={ }_{a} \Omega^{\alpha}{ }_{\beta}\left(\bar{\Psi}_{i}^{\beta}-2 \kappa \Pi_{+i b} \hat{\theta}_{-}^{b a} \bar{\Psi}_{a}^{\beta}\right) .
\end{aligned}
$$

Equations (5.16) and (5.20) are in full agreement with the results from Ref. [9] given by Eqs. (2.70) and (2.71).

The upper $D$ components of Eq. (5.6) produce the same result for T-dual background fields.

\subsection{T-dual R-R field strength $F^{\alpha \beta}$}

Using the relations ${ }_{a} \mathcal{H}=\mathcal{T}^{a} \mathcal{H} \mathcal{T}^{a}$ and ${ }_{a} J_{ \pm}=\mathcal{T}^{a} J_{ \pm}$we obtained the form of the NS-NS and NS-R T-dual background fields of type II superstring theory. But we know from the Buscher T-dualization procedure that the T-dual R$\mathrm{R}$ field strength ${ }_{a} F^{\alpha \beta}$ has the form given in Eq. (2.72). In this subsection we will derive this relation within the double space framework.

The R-R field strength $F^{\alpha \beta}$ appears in the action (2.13) coupled with the fermionic momenta $\pi_{\alpha}$ and $\bar{\pi}_{\alpha}$ along which we do not perform T-dualization. Therefore, we did not double these variables. It is an analog of the $i j$-term in approach of Refs. [27-29] where $x^{i}$ coordinates are not doubled. Consequently, as in [27-29] we should make some assumptions. Let us suppose that the fermionic term $L\left(\pi_{\alpha}, \bar{\pi}_{\alpha}\right)$ is symmetric under exchange of the R-R field strength $F^{\alpha \beta}$ with its T-dual ${ }_{a} F^{\alpha \beta}$

$L=e^{\frac{\Phi}{2}} \pi_{\alpha} F^{\alpha \beta} \bar{\pi}_{\beta}+e^{\frac{a \Phi}{2}}{ }_{a} \pi_{\alpha}{ }_{a} F^{\alpha \beta}{ }_{a} \bar{\pi}_{\beta} \equiv \mathcal{L}+{ }_{a} \mathcal{L}$,

for some $F^{\alpha \beta}$ and ${ }_{a} F^{\alpha \beta}$. This term should be invariant under the T-dual transformation

${ }_{a} \mathcal{L}=\mathcal{L}+\Delta \mathcal{L}$. 
Taking into account the fact that two successive T-dualizations are the identity transformation, we obtain from (5.22)

$\mathcal{L}={ }_{a} \mathcal{L}+{ }_{a} \Delta \mathcal{L}$.

Combining the last two relations we get

${ }_{a} \Delta \mathcal{L}=-\Delta \mathcal{L}$.

If $\Delta \mathcal{L}$ has the form $\Delta \mathcal{L}=\pi_{\alpha} \Delta^{\alpha \beta} \bar{\pi}_{\beta}$ and consequently ${ }_{a} \Delta \mathcal{L}={ }_{a} \pi_{\alpha{ }_{a} \Delta^{\alpha \beta}}{ }_{a} \bar{\pi}_{\beta}$, then with the help of the first relation of Eq. (2.48) we obtain the condition for $\Delta^{\alpha \beta}$

${ }_{a} \Delta^{\alpha \beta}=-\Delta^{\alpha \gamma}{ }_{a} \Omega_{\gamma}{ }^{\beta}$.

Therefore, we should find the combination of background fields with two upper spinor indices which under T-dualization transforms as in (5.25). Using the expression for the NS$\mathrm{R}$ fields (2.70) and the equation $\left({ }_{a} \hat{\theta}_{ \pm}\right)_{a b}=\frac{2}{\kappa} \Pi_{\mp a b}=$ $\frac{1}{\kappa^{2}}\left(\hat{\theta}_{ \pm}^{-1}\right)_{a b}$ [see the T-dual of (5.4) and (A.10)], it is easy to check that there are $D$ different solutions,

$\Delta_{d}^{\alpha \beta}=c \Psi_{a}^{\alpha} \hat{\theta}_{-}^{a b} \bar{\Psi}_{b}^{\beta}$,

where $d=1,2, \ldots D$ and $c$ is an arbitrary constant. Consequently, when we T-dualize $d$ dimensions $x^{a} \quad(a=$ $0,1, \ldots d-1)$, from $(5.22)$ we can conclude that the T-dual $\mathrm{R}-\mathrm{R}$ field strength has the form

$e^{\frac{a \Phi}{2}}{ }_{a} F^{\alpha \beta}=\left(e^{\frac{\Phi}{2}} F^{\alpha \gamma}+c \Psi_{a}^{\alpha} \hat{\theta}_{-}^{a b} \bar{\Psi}_{b}^{\gamma}\right){ }_{a} \Omega_{\gamma}{ }^{\beta}$.

For $c=4 \kappa$ we obtain the agreement with Eq. (2.72). Note that the fermionic term $L_{d}\left(\pi_{\alpha}, \bar{\pi}_{\alpha}\right)$ depends on $d$, the number of directions along which we perform T-duality, just in Refs. [27-29].

\section{Conclusion}

In this article we showed that the new interpretation of the bosonic T-dualization procedure in the double space formalism offered in $[35,36]$ is also valid in the case of type II superstring theory. We used the ghost free action of type II superstring theory in a pure spinor formulation in the approximation of quadratic terms and constant background fields. One can obtain this action from the action (2.7), which could be considered as an expansion in powers of fermionic coordinates. In the first part of the analysis we neglect all terms in the action containing powers of $\theta^{\alpha}$ and $\bar{\theta}^{\alpha}$. This approximation is justified by the fact that the action is a result of an iterative procedure in which every step results from the previous one. Later, when we discuss proper fermionic variables, taking higher power terms we restore supersymmetric invariants $\left(\Pi_{ \pm}^{\mu}, d_{\alpha}, \bar{d}_{\alpha}\right)$ as variables instead of $\partial_{ \pm} x^{\mu}, \pi_{\alpha}$ and $\bar{\pi}_{\alpha}$.

We introduced the double space coordinate $Z^{M}=$ $\left(x^{\mu}, y_{\mu}\right)$ adding to all bosonic initial coordinates, $x^{\mu}$, the
T-dual ones, $y_{\mu}$. Then we rewrote the T-dual transformation laws (3.8) in terms of double space variables (3.12) introducing the generalized metric $\mathcal{H}_{M N}$ and the current $J_{ \pm M}$. The generalized metric depends only on the NS-NS background fields of the initial theory. The current $J_{ \pm M}$ contains fermionic momenta $\pi_{\alpha}$ and $\bar{\pi}_{\alpha}$, along which we do not make a T-dualization, and it depends also on NS-R background fields. The R-R background fields do not appear in T-dual transformation laws.

The coordinate index $\mu$ is split in $a=(0,1, \ldots d-1)$ and $i=(d, d+1, \ldots D-1)$, where index $a$ marks subsets of the initial and T-dual coordinates, $x^{a}$ and $y_{a}$, along which we make T-dualization. T-dualization is realized as permutation of the subsets $x^{a}$ and $y_{a}$ in the double space coordinate $Z^{M}$. The main require is that $\mathrm{T}$-dual double space coordinates ${ }_{a} Z^{M}=\left(\mathcal{T}^{a}\right)^{M}{ }_{N} Z^{N}$ satisfy the transformation law of the same form as the initial coordinates $Z^{M}$. From this condition we found the T-dual generalized metric ${ }_{a} \mathcal{H}_{M N}$ and the Tdual current ${ }_{a} J_{ \pm M}$. Because the initial and T-dual theory are physically equivalent, ${ }_{a} \mathcal{H}_{M N}$ and ${ }_{a} J_{ \pm M}$ should have the same form as the initial ones, $\mathcal{H}$ and $J_{ \pm M}$, but in terms of the T-dual background fields. It produces the form of the NSNS and NS-R T-dual background fields in terms of the initial ones which are in full accordance with the results obtained by the Buscher T-dualization procedure $[9,10]$.

The supersymmetry case is not a simple generalization of the bosonic one, but it requires some new interesting steps. The origin of the problem is the different T-duality transformations of the world-sheet chirality sectors. It produces two possible sets of vielbeins in the T-dual theory with the same T-dual metric. These vielbeins are related by a particular local Lorentz transformation which depends on T-duality transformation and of which the determinant is $(-1)^{d}$, where $d$ is the number of T-dualized coordinates. Therefore, when we perform T-dualization along an odd number of coordinates then such transformation contains a parity transformation. Consistency of T-duality with supersymmetry requires changing one of two spinor sectors. We redefine the bar spinor coordinates, ${ }_{a} \bar{\theta} \rightarrow \dot{\bullet}_{a} \bar{\theta}^{\alpha}={ }_{a} \Omega^{\alpha}{ }_{\beta} \bar{\theta}^{\beta}$, and the variable ${ }_{a} \bar{\pi}_{\alpha}$, ${ }_{a} \bar{\pi}_{\alpha} \rightarrow{ }_{a} \bar{\pi}_{\alpha}={ }_{a} \Omega_{\alpha}{ }^{\beta} \bar{\pi}_{\beta}$. As a consequence the bar NS$\mathrm{R}$ and R-R background fields include ${ }_{a} \Omega$ in their T-duality transformations. For an odd number of coordinates $d$ along which T-dualization is performed, ${ }_{a} \Omega$ changes the chirality of the bar gravitino $\bar{\Psi}_{\mu}^{\alpha}$ and the chirality condition for $F^{\alpha \beta}$. We need it to relate type IIA and type IIB theories.

The transformation law (3.12) induces the consistency condition which can be considered as equation of motion of the double space action (3.21). It contains an arbitrary term depending on the undualized variables $L\left(\pi_{\alpha}, \bar{\pi}_{\alpha}\right)$. This is in analogy with the term $\partial_{+} x^{i} \Pi_{+i j} \partial_{-} x^{j}$ in the approach presented in Refs. [27-29]. Therefore, to obtain the T-dual transformation of the $\mathrm{R}-\mathrm{R}$ field strength $F^{\alpha \beta}$ we should make some additional assumptions. Supposing that the term 
$L\left(\pi_{\alpha}, \bar{\pi}_{\alpha}\right)$ is T-dual invariant and taking into account that two successive T-dualizations act as the identity operator, we found the form of the T-dual R-R field strength up to one arbitrary constant $c$. For $c=4 \kappa$ we get the T-dual R-R field strength ${ }_{a} F^{\alpha \beta}$ as in the Buscher procedure [9].

A T-duality transformation of the R-R field strength $F^{\alpha \beta}$ has two contributions in the form of square roots. The contribution of the dilaton produces the term $\sqrt{\left|\prod_{i=1}^{d} G_{a_{i} a_{i}}\right|}$. On the other hand the contribution of the spinorial representation of a Lorentz transformation ${ }_{a} \Omega$ contains the same expression without the absolute value $i \sqrt{\prod_{i=1}^{d} G_{a_{i} a_{i}}}$. Therefore, the Tdual R-R field strength ${ }_{a} F^{\alpha \beta}$, besides a rational expression, contains the expression $i d \sqrt{\operatorname{sign}\left(\prod_{i=1}^{d} G_{a_{i} a_{i}}\right)}$ (2.89). If we T-dualize along the time-like direction $\left(G_{00}>0\right)$, the square root does not produce an imaginary unit $i$, not canceling the one in front of the square root. Therefore, T-dualization along the time-like direction maps type II superstring theories to type II $^{\star}$ ones [37].

The successive T-dualizations make a group called the Tduality group. In the case of type II superstring T-duality, transformations are performed by the same matrices $\mathcal{T}^{a}$ as in the bosonic string case $[35,36]$. Consequently, the corresponding T-duality group is the same.

If we want to find a T-dual transformation of $F^{\alpha \beta}$ without any assumptions, we should follow the approach of $[35,36]$ and, besides all bosonic coordinates $x^{\mu}$, double also all fermionic variables $\pi_{\alpha}$ and $\bar{\pi}_{\alpha}$. In other words, besides bosonic T-duality we should also consider fermionic T-duality [53-59].

Open Access This article is distributed under the terms of the Creative Commons Attribution 4.0 International License (http://creativecomm ons.org/licenses/by/4.0/), which permits unrestricted use, distribution, and reproduction in any medium, provided you give appropriate credit to the original author(s) and the source, provide a link to the Creative Commons license, and indicate if changes were made.

Funded by SCOAP ${ }^{3}$.

\section{Appendix A: Block-wise expressions for background fields}

In order to simplify notation we will introduce notations for the component fields following Ref. [35].

For block-wise matrices there is a rule for inversion,

$$
\begin{aligned}
& \left(\begin{array}{ll}
A & B \\
C & D
\end{array}\right)^{-1} \\
& =\left(\begin{array}{cc}
\left(A-B D^{-1} C\right)^{-1} & -A^{-1} B\left(D-C A^{-1} B\right)^{-1} \\
-D^{-1} C\left(A-B D^{-1} C\right)^{-1} & \left(D-C A^{-1} B\right)^{-1}
\end{array}\right) .
\end{aligned}
$$

For the metric tensor and the Kalb-Ramond background fields we define

$G_{\mu \nu}=\left(\begin{array}{cc}\tilde{G}_{a b} & G_{a j} \\ G_{i b} & \bar{G}_{i j}\end{array}\right) \equiv\left(\begin{array}{cc}\tilde{G} & G^{T} \\ G & \bar{G}\end{array}\right)$

and

$B_{\mu \nu}=\left(\begin{array}{ll}\tilde{b}_{a b} & b_{a j} \\ b_{i b} & \bar{b}_{i j}\end{array}\right) \equiv\left(\begin{array}{cc}\tilde{b} & -b^{T} \\ b & \bar{b}\end{array}\right)$.

We also define the notation for inverse of the metric,

$\left(G^{-1}\right)^{\mu \nu}=\left(\begin{array}{ll}\tilde{\gamma}^{a b} & \gamma^{a j} \\ \gamma^{i b} & \bar{\gamma}^{i j}\end{array}\right) \equiv\left(\begin{array}{cc}\tilde{\gamma} & \gamma^{T} \\ \gamma & \bar{\gamma}\end{array}\right)$,

and for the effective metric

$G_{\mu \nu}^{E}=G_{\mu \nu}-4 B_{\mu \rho}\left(G^{-1}\right)^{\rho \sigma} \quad B_{\mu \nu}=\left(\begin{array}{cc}\tilde{g}_{a b} & g_{a j} \\ g_{i b} & \bar{g}_{i j}\end{array}\right) \equiv\left(\begin{array}{cc}\tilde{g} & g^{T} \\ g & \bar{g}\end{array}\right)$.

Note that because $G^{\mu \nu}$ is the inverse of $G_{\mu \nu}$ we have

$\gamma=-\bar{G}^{-1} G \tilde{\gamma}=-\bar{\gamma} G \tilde{G}^{-1}$,

$\gamma^{T}=-\tilde{G}^{-1} G^{T} \bar{\gamma}=-\tilde{\gamma} G^{T} \bar{G}^{-1}$,

$\tilde{\gamma}=\left(\tilde{G}-G^{T} \bar{G}^{-1} G\right)^{-1}, \quad \bar{\gamma}=\left(\bar{G}-G \tilde{G}^{-1} G^{T}\right)^{-1}$,

$\tilde{G}^{-1}=\tilde{\gamma}-\gamma^{T} \bar{\gamma}^{-1} \gamma, \quad \bar{G}^{-1}=\bar{\gamma}-\gamma \tilde{\gamma}^{-1} \gamma^{T}$.

It is also useful to introduce a new notation for the expression

$\left(B G^{-1}\right)_{\mu}{ }^{\nu}=\left(\begin{array}{cc}\tilde{b} \tilde{\gamma}-b^{T} \gamma & \tilde{b} \gamma^{T}-b^{T} \bar{\gamma} \\ b \tilde{\gamma}+\bar{b} \gamma & b \gamma^{T}+\bar{b} \bar{\gamma}\end{array}\right) \equiv\left(\begin{array}{cc}\tilde{\beta} & \beta_{1} \\ \beta_{2} & \bar{\beta}\end{array}\right)$.

We denote by a hat expressions similar to the effective metric (A.5) and non-commutativity parameters but with all contributions from the $a b$ subspace,

$\hat{g}_{a b}=\left(\tilde{G}-4 \tilde{b} \tilde{G}^{-1} \tilde{b}\right)_{a b}, \quad \hat{\theta}^{a b}=-\frac{2}{\kappa}\left(\hat{g}^{-1} \tilde{b} \tilde{G}^{-1}\right)^{a b}$.

Note that $\hat{g}_{a b} \neq \tilde{g}_{a b}$ because $\tilde{g}_{a b}$ is a projection of $g_{\mu \nu}$ on the subspace $a b$. It is extremely useful to introduce background field combinations,

$$
\begin{aligned}
\Pi_{ \pm a b} & =B_{a b} \pm \frac{1}{2} G_{a b} \\
\hat{\theta}_{ \pm}^{a b} & =-\frac{2}{\kappa}\left(\hat{g}^{-1} \tilde{\Pi}_{ \pm} \tilde{G}^{-1}\right)^{a b}=\hat{\theta}^{a b} \mp \frac{1}{\kappa}\left(\hat{g}^{-1}\right)^{a b}
\end{aligned}
$$

which are inverses to each other

$\hat{\theta}_{ \pm}^{a c} \Pi_{\mp c b}=\frac{1}{2 \kappa} \delta_{b}^{a}$.

The quantity $A_{a b}$ is defined as

$A_{a b}=\left(\tilde{g}-4 \beta_{1} \bar{\gamma}^{-1} \beta_{1}^{T}\right)_{a b}$.

One can prove the relation [35]

$\left(\tilde{g}^{-1} \beta_{1} D^{-1}\right)^{a}{ }_{i}=\left(\hat{g}^{-1} \beta_{1} \bar{\gamma}^{-1}\right)^{a}{ }_{i}$, 
where $D^{i j}$ is defined in Eq. (3.21) of [35],

$$
\begin{aligned}
& D^{i j}=\left(\bar{\gamma}-4 \beta_{1}^{T} \tilde{g}^{-1} \beta_{1}\right)^{i j}, \\
& \quad\left(D^{-1}\right)_{i j}=\left(\bar{\gamma}^{-1}+4 \bar{\gamma}^{-1} \beta_{1}^{T} A^{-1} \beta_{1} \bar{\gamma}^{-1}\right)_{i j} .
\end{aligned}
$$

\section{References}

1. K. Becker, M. Becker, J. Schwarz, String Theory and M-Theory: A Modern Introduction (Cambridge University Press, Cambridge, 2007)

2. B. Zwiebach, A First Course in String Theory (Cambridge University Press, Cambridge, 2004)

3. J. Polchinski, String Theory, vol. II (Cambridge University Press, Cambridge, 1998)

4. T. Buscher, Phys. Lett. B 194, 59 (1987)

5. T. Buscher, Phys. Lett. 201, 466 (1988)

6. M. Roček, E. Verlinde, Nucl. Phys. B 373, 630 (1992)

7. A. Giveon, M. Porrati, E. Rabinovici, Phys. Rep. 244, 77 (1994)

8. E. Alvarez, L. Alvarez-Gaume, J. Barbon, Y. Lozano, Nucl. Phys. B 415, 71 (1994)

9. B. Nikolić, B. Sazdović, Nucl. Phys. B 836, 100 (2010)

10. D.S. Berman, D.C. Thompson, Phys. Rep. 566, 1-60 (2014)

11. D. Lust, JHEP 12, 084 (2010)

12. D. Andriot, M. Larfors, D. Luest, P. Patalong, JHEP 06, 021 (2013)

13. D. Andriot, O. Hohm, M. Larfors, D. Lust, P. Patalong, Phys. Rev. Lett. 108, 261602 (2012)

14. D. Lüst, in Proceedings of the Corfu Summer Institute 2011 School and Workshops on Elementary Particle Physics and Gravity. September 4-18, 2011. Corfu, Greece. arXiv:1205.0100 [hep-th]

15. R. Blumenhagen, A. Deser, D. Luest, E. Plauschinn, F. Rennecke, J. Phys. A 44, 385401 (2011)

16. C. Condeescu, I. Florakis, D. Luest, JHEP 04, 121 (2012)

17. J. Shelton, W. Taylor, B. Wecht, JHEP 10, 085 (2005)

18. A. Dabholkar, C. Hull, JHEP 05, 009 (2006)

19. Lj. Davidović, B. Sazdović, EPJ C 74, 2683 (2014)

20. Lj. Davidović, B. Nikolić, B. Sazdović, EPJ C 75, 576 (2015)

21. Lj. Davidović, B. Nikolić, B. Sazdović, EPJ C 74, 2734 (2014)

22. M. Duff, Nucl. Phys. B 335, 610 (1990)

23. A.A. Tseytlin, Phys. Lett. B 242, 163 (1990)

24. A.A. Tseytlin, Nucl. Phys. B 350, 395 (1991)

25. W. Siegel, Phys. Rev. D 48, 2826 (1993)

26. W. Siegel, Phys. Rev. D 47, 5453 (1993)

27. C.M. Hull, JHEP 10, 065 (2005)

28. C.M. Hull, JHEP 10, 057 (2007)
29. C.M. Hull, JHEP 07, 080 (2007)

30. D.S. Berman, M. Cederwall, M.J. Perry, JHEP 09, 066 (2014)

31. D.S. Berman, C.D.A. Blair, E. Malek, M.J. Perry, Int. J. Mod. Phys. A 29(15), 1450080 (2014)

32. C.D.A. Blair, E. Malek, A.J. Routh, Class. Quantum Gravity 31(20), 205011 (2014)

33. C.M. Hull, R.A. Reid-Edwards, JHEP 09, 014 (2009)

34. O. Hohm, B. Zwiebach, JHEP 11, 075 (2014)

35. B. Sazdović, T-duality as coordinates permutation in double space. arXiv:1501.01024. doi:10.1088/1674-1137/41/5/053101

36. B. Sazdović, JHEP 08, 055 (2015)

37. C.M. Hull, JHEP 07, 021 (1998)

38. N. Berkovits, ICTP Lectures on Covariant Quantisation of Superstrings, arXiv:hep-th/0209059

39. P.A. Grassi, G. Policastro, P. van Nieuwenhuizen, JHEP 10, 054 (2002)

40. P.A. Grassi, G. Policastro, P. van Nieuwenhuizen, JHEP 11, 004 (2002)

41. P.A. Grassi, G. Policastro, P. van Nieuwenhuizen, Adv. Theor. Math. Phys. 7, 499 (2003)

42. P.A. Grassi, G. Policastro, P. van Nieuwenhuizen, Phys. Lett. B 553, 96 (2003)

43. J. de Boer, P.A. Grassi, P. van Nieuwenhuizen, Phys. Lett. B 574, 98 (2003)

44. N. Berkovits, P. Howe, Nucl. Phys. B 635, 75 (2002)

45. P.A. Grassi, L. Tamassia, JHEP 07, 071 (2004)

46. S.F. Hassan, Nucl. Phys. B 568, 145 (2000)

47. R. Benichou, G. Policastro, J. Troost, Phys. Lett. B 661, 129 (2008)

48. M.J. Duff, TASI Lectures on Branes, Black Holes and Anti-de Sitter Space, arXiv:hep-th/9912164v2

49. M.J. Duff, R.R. Khuri, J.X. Lu, Phys. Rep. 259, 213 (1995)

50. E. Kiritsis, Introduction to Superstring Theory (Leuven University Press, Leuven, 1998). arXiv:hep-th/9709062

51. B. Nikolić, B. Sazdović, Phys. Lett. B 666, 400 (2008)

52. D.C. Thompson, T-Duality Invariant Approaches to String Theory, $\mathrm{PhD}$ thesis. arXiv: 1012.4393

53. N. Berkovits, J. Maldacena, JHEP 09, 062 (2008)

54. K. Sfetsos, K. Siampos, D.C. Thompson, Class. Quantum Gravity 28, 055010 (2011)

55. I. Bakhmatov, D.S. Berman, Nucl. Phys. B 832, 89-108 (2010)

56. N. Beisert, R. Ricci, A.A. Tseytlin, M. Wolf, Phys. Rev. D 78, 126004 (2008)

57. R. Ricci, A.A. Tseytlin, M. Wolf, JHEP 12, 082 (2007)

58. B. Nikolić, B. Sazdović, Phys. Rev. D 84, 065012 (2011)

59. B. Nikolić, B. Sazdović, JHEP 06, 101 (2012)

60. A. Giveon, M. Roček, Nucl. Phys. B 421, 173 (1994) 